\title{
Self-expandable metal stents for obstructing colonic and extracolonic cancer: European Society of Gastrointestinal Endoscopy (ESGE) Guideline - Update 2020
}

\section{(3) ESGE}

Authors

Jeanin E. van Hooft ${ }^{1}$, Joyce V. Veld ${ }^{1,2}$, Dirk Arnold ${ }^{3}$, Regina G.H. Beets-Tan ${ }^{4}$, Simon Everett ${ }^{5}$, Martin Götz ${ }^{6}$, Emo E. van Halsema ${ }^{1}$, James Hill7 , Gianpiero Manes ${ }^{8}$, Soren Meisner ${ }^{9}$, Eduardo Rodrigues-Pinto ${ }^{10}$, Charles Sabbagh ${ }^{11}$, Jo Vandervoort ${ }^{12}$, Pieter ]. Tanis ${ }^{2}$, Geoffroy Vanbiervliet ${ }^{13}$, Alberto Arezzo ${ }^{14}$

Institutions

1 Department of Gastroenterology and Hepatology, Amsterdam UMC, University of Amsterdam, Cancer Center Amsterdam, The Netherlands

2 Department of Surgery, Amsterdam UMC, University of Amsterdam, Cancer Center Amsterdam, The Netherlands

3 Department of Oncology, Hematology and Palliative Care, Asklepios Tumourzentrum Hamburg, Hamburg, Germany

4 Department of Radiology, The Netherlands Cancer Institute, Amsterdam, The Netherlands

5 Department of Gastroenterology and Hepatology, Leeds Teaching Hospital NHS Trust, Leeds, United Kingdom

6 Department of Gastroenterology/Oncology, Klinikum Sindelfingen-Böblingen, Kliniken Böblingen, Böblingen, Germany

7 Department of Surgery, Manchester University NHS FT, Manchester, United Kingdom

8 Department of Gastroenterology and Endoscopy, Guido Salvini Hospital, Garbagnate Milanese/Rho, Milan, Italy

9 Endoscopy Unit, Digestive Disease Center, Bispebjerg University Hospital, Copenhagen, Denmark

10 Department of Gastroenterology, Centro Hospitalar São João, Porto, Portugal

11 Department of Digestive and Oncological Surgery, University Hospital of Amiens, France

12 Department of Gastroenterology, Onze-LieveVrouwziekenhuis, Aalst, Belgium

13 Department of Gastroenterology, Centre Hospitalier Universitaire de l'Archet, Pôle digestif, Nice, France

14 Department of Surgical Sciences, Università di Torino, Torino, Italy
Bibliography

DOI https://doi.org/10.1055/a-1140-3017

Published online: 7.4.2020 | Endoscopy 2020; 52: 389-407

(c) Georg Thieme Verlag KG Stuttgart · New York

ISSN 0013-726X

Corresponding author

Jeanin E. van Hooft, MD PhD MBA, Department of Gastroenterology and Hepatology, C2-115, Amsterdam University Medical Center, location AMC, Meibergdreef 9, 1105 AZ, Amsterdam, The Netherlands Fax: +31206917033

j.e.vanhooft@amsterdamumc.nl

\# Appendix 1s-3s

Online content viewable at:

https://doi.org/10.1055/a-1140-3017

\section{MAIN RECOMMENDATIONS}

The following recommendations should only be applied after a thorough diagnostic evaluation including a contrast-enhanced computed tomography (CT) scan.

1 ESGE recommends colonic stenting to be reserved for patients with clinical symptoms and radiological signs of malignant large-bowel obstruction, without signs of perforation. ESGE does not recommend prophylactic stent placement.

Strong recommendation, low quality evidence.

2 ESGE recommends stenting as a bridge to surgery to be discussed, within a shared decision-making process, as a treatment option in patients with potentially curable left-sided obstructing colon cancer as an alternative to emergency resection.

Strong recommendation, high quality evidence.

3 ESGE recommends colonic stenting as the preferred treatment for palliation of malignant colonic obstruction. Strong recommendation, high quality evidence. 
4 ESGE suggests consideration of colonic stenting for malignant obstruction of the proximal colon either as a bridge to surgery or in a palliative setting.

Weak recommendation, low quality evidence.

5 ESGE suggests a time interval of approximately 2 weeks until resection when colonic stenting is performed as a bridge to elective surgery in patients with curable left-sided colon cancer.

Weak recommendation, low quality evidence.
6 ESGE recommends that colonic stenting should be performed or directly supervised by an operator who can demonstrate competence in both colonoscopy and fluoroscopic techniques and who performs colonic stenting on a regular basis.

Strong recommendation, low quality evidence.

7 ESGE suggests that a decompressing stoma as a bridge to elective surgery is a valid option if the patient is not a candidate for colonic stenting or when stenting expertise is not available.

Weak recommendation, low quality evidence.

\section{SOURCE AND SCOPE}

This Guideline is an official statement of the European Society of Gastrointestinal Endoscopy (ESGE). It is a revision of the previously published 2014 Guideline addressing the role of self-expandable metal stents for obstructing colonic and extracolonic cancer.

\section{Introduction}

Colorectal cancer is one of the most common cancers worldwide, particularly in the economically developed world [1]. Large-bowel obstruction caused by advanced colonic cancer occurs in $8 \%-13 \%$ of colonic cancer patients [ $2-4]$. The management of this severe clinical condition has been controversial [5]. Over the last decade, many articles have been published on the subject of colonic stenting for malignant colonic obstruction, including randomized controlled trials (RCTs) and systematic reviews. Thereby, the role of self-expandable metal stents (SEMSs) in the treatment of malignant colonic obstruction has become better defined. This evidence- and consensus-based clinical guideline has been developed by the European Society of Gastrointestinal Endoscopy (ESGE). It is an update of the previously published guideline [6], and aims to put into perspective the new evidence that has become available over the last 5 years and to provide statements regarding the use of SEMS in the treatment of malignant colonic obstruction.

With the exception of one trial [7], all published RCTs on colonic stenting for malignant obstruction excluded rectal cancers, which were usually defined as within 8 to $10 \mathrm{~cm}$ of the anal verge, and colonic cancers proximal to the splenic flexure. Rectal stenting is often avoided because of the presumed association with complications such as pain, tenesmus, incontinence, and stent migration. Proximal colonic obstruction is generally managed with primary surgery, although there are no RCTs to support this assumption. Because of the aforementioned limitations, unless indicated otherwise, the recommendations in this Guideline only apply to left-sided colon cancer arising from the rectosigmoid colon, sigmoid colon, descend- ing colon, and splenic flexure, while excluding rectal cancers and those proximal to the splenic flexure, and other causes of colonic obstruction including extracolonic obstruction.

\section{Methods}

ESGE commissioned this Guideline and appointed a guideline leader (J.v.H.), who invited the listed authors to participate in the project development. The key questions were prepared by the coordinating team (J.V. and J.v.H.) and then approved by the other members. The coordinating team formed task force subgroups, each with its own leader, and divided the key topics among these task forces (see Appendix 1s, online-only Supplementary Material).

Each task force performed a systematic literature search to prepare evidence-based and well-balanced statements on their assigned key questions. The coordinating team independently

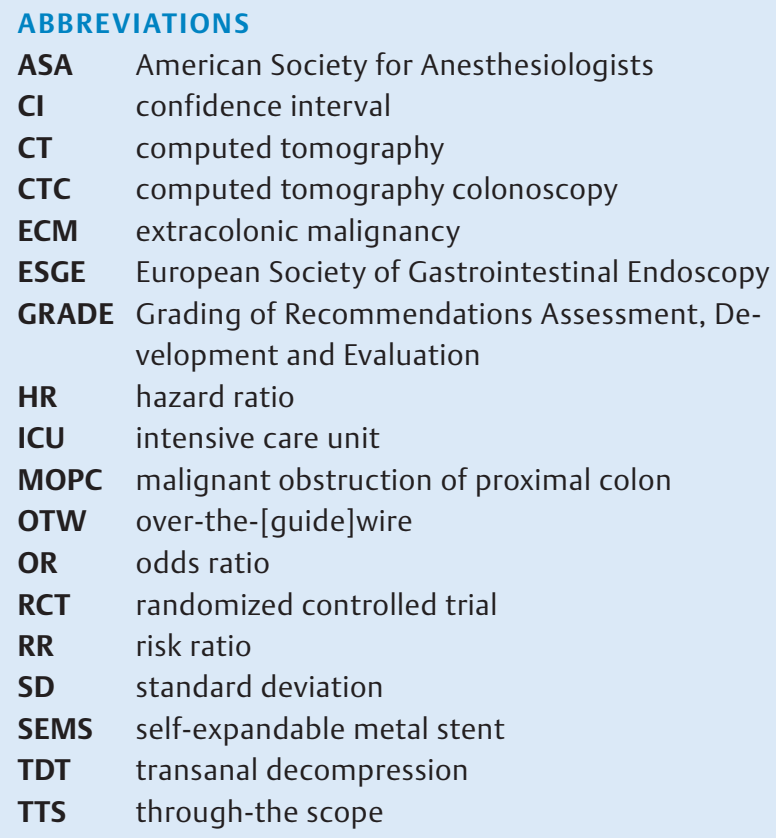


performed systematic literature searches with the assistance of a librarian. The Medline, EMBASE, and Cochrane Library databases were searched including at a minimum the following key words: colon, cancer, malignancy or neoplasm, obstruction, and stents. All articles studying the use of colonic stenting for malignant large-bowel obstruction were selected by title or abstract. After further exploration of the content, articles containing relevant data were then included and summarized in the literature tables of the key topics (see Appendix 2s, Tables 1s-5s). All selected articles were graded by the level of evidence and strength of recommendation according to the Grading of Recommendations Assessment, Development and Evaluation (GRADE) system [8]. The literature searches were updated until July 2019.

Each task force proposed statements on their assigned key questions which were discussed and voted on during the plenary meeting held in September 2019, Amsterdam, The Netherlands. An overview of the statements of the previous guideline published in 2014 [6] versus the updated statements was created (Appendix 3s). In October 2019, a draft prepared by the coordinating team was sent to all group members. After agreement of all members had been obtained, the manuscript was reviewed by two external reviewers and was sent for further comments to the ESGE national societies and individual members. After this, the manuscript was submitted to the journal Endoscopy for publication. All authors agreed on the final revised manuscript.

This Guideline was issued in 2020 and will be considered for review in 2025 or sooner if new and relevant evidence becomes available. Any updates to the Guideline in the interim will be noted on the ESGE website: http://www.esge.com/esgeguidelines.html.

\section{Recommendations and statements}

\section{General considerations before colonic stenting}

\section{RECOMMENDATION}

ESGE recommends colonic stenting to be reserved for patients with clinical symptoms and radiological signs of malignant large-bowel obstruction, without signs of perforation. ESGE does not recommend prophylactic stent placement.

Strong recommendation, low quality evidence.

Colonic stenting is indicated only in those patients with both obstructive symptoms and radiological findings suspicious of malignant large-bowel obstruction. Obstruction is a predominant complication of the tumor in patients treated primarily with chemotherapy because of metastasized colorectal cancer at diagnosis [9]. Colonoscopic nontraversability, i. e., the inability to advance the scope beyond the tumor, has been suggested as a risk factor for the development of symptomatic bowel obstruction during treatment with primary chemotherapy [1012]. Nevertheless, prophylactic stenting for patients with colo- nic malignancy without evidence of symptomatic obstruction is strongly discouraged because of the potential risks associated with colonic stenting.

The only absolute contraindication for colonic stenting is perforation. In addition, colonic stenting is less successful in patients with peritoneal metastases and tumors close to the anal verge $(<5 \mathrm{~cm})$, the latter because of tenesmus [13-15]. There is no high quality literature to grade the severity of the obstruction. A Japanese group developed the ColoRectal Obstruction Scoring System (CROSS) aimed at aiding the evaluation of severity of colonic obstruction [16]. Studies comparing the outcomes of total and subtotal obstruction used different definitions (e.g. based on symptoms, radiologic, or endoscopic findings) and reported inconsistent outcomes. Increasing age and American Society of Anesthesiologists (ASA) classification $\geq$ III do not affect stenting outcome (i.e., clinical success and complications) in several observational studies [17-22], although these are well-known risk factors for postoperative mortality after surgical treatment of large-bowel obstruction [23-25].

\section{RECOMMENDATION}

ESGE recommends performing contrast-enhanced computed tomography (CT) scan when malignant colonic obstruction is suspected.

Strong recommendation, low quality evidence.

When malignant colonic obstruction is suspected, contrastenhanced CT imaging is recommended because it can diagnose obstruction (sensitivity $96 \%$, specificity $93 \%$ ), define the level of the stenosis in $94 \%$ of cases, accurately identify the etiology in $81 \%$ of cases, and provide correct local and distal staging in the majority of patients $[5,26]$. When $\mathrm{CT}$ is inconclusive about the etiology of the obstructing lesion, colonoscopy may be helpful to evaluate the exact cause of the stenosis.

Regarding cecal pneumatosis on $\mathrm{CT}$, one small retrospective study of 10 patients has been published concluding that despite CT findings of cecal pneumatosis, the cecum was deemed to be viable intraoperatively in all patients [27]. The authors concluded that cecal pneumatosis alone is not a reliable predictor of cecal ischemia in patients presenting with acute malignant large-bowel obstruction and that colonic stenting should not be precluded in these patients. However, as literature is very scarce on this subject, no recommendations regarding cecal pneumatosis and colonic stenting can be made.

\section{RECOMMENDATION}

ESGE recommends examination of the remaining colon to exclude synchronous pathology in patients with potentially curable colonic cancer, either before or no more than 6 months after alleviation of the colonic obstruction. Strong recommendation, low quality evidence. 
The risk of proximal neoplasia increases in the presence of distal lesions. European studies, including three that are population-based, show that synchronous colorectal tumors occur in $3 \%-4 \%$ of patients diagnosed with colorectal cancer [28-31]. Since 2014, several studies have assessed preoperative through-the-stent colonoscopy in patients with stenosing colorectal cancer [32-35]. The majority of patients underwent bowel cleansing with polyethylene glycol $[33,34]$. Completed preoperative colonoscopy rates ranged from $62.5 \%$ to $96.6 \%$ [32 - 34], with the lowest rate increasing to $87.5 \%$ when an additional gastroscope was used [34]. Incomplete colonoscopy was mostly related to the degree of stent expansion. Synchronous colorectal cancers were diagnosed in $0 \%-17.9 \%$ of cases and adenomatous lesions in $29.4 \%-60.7 \%$ [32-34]. One study described a patient having subclinical subdiaphragmatic free air (2.1\%) and eight having self-limited minor bleeding (16.6\%) after the procedure [34].

CT colonography is at least as effective as colonoscopy in identifying colonic lesions (sensitivity $97.56 \%$ vs. $92.68 \%$, negative predictive value $93.75 \%$ vs. $83.3 \%$ ), more frequently allowing complete colon visualization (100\% vs. 62\%) [36]. However, the clinical impact of CT colonography in stenosing colorectal cancer is debatable, as it correctly changes the primary surgical plan in only $1.9 \%$ of patients. Moreover, there is a risk of false-positive results [37]. Positron emission tomography (PET)/CT has a high sensitivity and negative predictive value in recognizing synchronous lesions in patients with obstructive colorectal cancer. It would thus allow definition of the correct surgical plan [38-40], although it is infrequently used in clinical practice.

In general, exploration of the remaining colon is advisable in patients with distal stenosing colorectal cancer, but no studies have specifically evaluated its ideal timing. Colon exploration may be performed either before or no more than 6 months after alleviation of the colonic obstruction. Both conventional colonoscopy and CT colonography are feasible, but some risk of complications and potential spread of tumor cells through endoscopic manipulation may be related to preoperative colonoscopy.

\section{RECOMMENDATION}

ESGE recommends that colonic stenting for diverticular disease should be avoided.

Strong recommendation, low quality evidence.

\section{RECOMMENDATION}

ESGE recommends to take endoscopic biopsies of an obstructing tumor; however pathological confirmation of malignancy should not persistently be pursued in an urgent setting, such as during stent placement for acute colonic obstruction.

Strong recommendation, low quality evidence.
When a malignancy is suspected after diagnostic studies, a small number of patients will have a benign cause of obstruction. Two RCTs comparing SEMS as a bridge to surgery versus emergency surgery in patients with left-sided malignant obstruction reported benign obstructive lesions in $4.6 \%(3 / 65)$ [41] and $8.2 \%(8 / 98)$ [42] of the randomized patients. These benign colonic lesions that mimic malignancy are usually due to diverticular disease. Further evidence of the difficulty of this distinction is also reflected by a systematic review, showing a $2.1 \%$ prevalence of underlying adenocarcinoma of the colon in 771 patients in whom acute diverticulitis was diagnosed through CT imaging [43]. Stent placement in active diverticular inflammation is associated with a risk of perforation and should therefore be avoided [44]. Additionally, pathological confirmation of malignancy before emergency stent placement is often not feasible and is not required prior to colonic stenting. However, endoscopic biopsy for confirmation of malignancy should preferably be obtained during the stent placement procedure, as it may modify the further management of the stented patient [45-47]. In cases where pathology shows benign disease, one has to consider the possibility of sampling error. Otherwise, early resection of a suspected benign obstruction might be indicated.

\section{RECOMMENDATION}

ESGE suggests that patients with a colonic obstruction should receive preparation with an enema to clean the colon distal to the stenosis in order to facilitate stricture visualization and stent placement.

Weak recommendation, low quality evidence.

\section{RECOMMENDATION}

ESGE does not recommend antibiotic prophylaxis specifically for colonic stenting.

Strong recommendation, low quality evidence.

Symptomatic bowel obstruction is a relative contraindication to oral bowel cleansing. The majority of studies do not report on performance of bowel preparation or cleansing enemas before stent placement. Among studies published since 2014, use of a cleansing enema before stent placement was mentioned in only $16.4 \%$, and in $1.2 \%$ oral bowel preparation was performed according to tolerance or in the circumstances of incomplete obstruction. A post hoc analysis of a prospective multicenter study showed that preparation with a cleansing enema facilitated stent placement, resulting in slightly fewer procedures exceeding a procedure time beyond the 75 th percentile $(23.8 \%$ vs. $28.9 \%$, odds ratio [OR] $0.5, P<0.01$ ) [48].

Antibiotic prophylaxis before colonic stenting in patients with malignant colonic obstruction is not indicated because the risk of fever and bacteremia after stent insertion is very low. One prospective study analyzed 64 patients with colorectal 
cancer who underwent a stent procedure [49]. Four of 64 patients $(6.3 \%)$ had a positive post-stenting blood culture and none of the patients developed symptoms of infection within 48 hours following stent placement. Prolonged procedure time was associated with transient bacteremia (36 vs. 16 minutes, $P<$ 0.01). One other retrospective series of 233 patients undergoing colonic stenting for malignant obstruction described that blood cultures had been drawn for unspecified reasons in 30 patients within 2 weeks after stent placement, showing bacteremia/fever in seven patients (3\%), which was reported as a minor complication [21]. A propensity score-matched analysis of prophylactic antibiotics for colonic stenting showed no significant differences in post-SEMS insertion infectious complications, such as fever, bacteremia, and systemic inflammatory response syndrome [50].

\section{RECOMMENDATION}

ESGE recommends that colonic stenting should be performed or directly supervised by an operator who can demonstrate competence in both colonoscopy and fluoroscopic techniques and who performs colonic stenting on a regular basis.

Strong recommendation, low quality evidence.

Several noncomparative studies addressed the learning curve of single endoscopists performing colonic stenting [22, $51-54]$. In most of these studies, it was mentioned that the endoscopists were experienced in colonoscopy. Two studies showed an increase in technical success and a decrease in the number of stents used per procedure after performance of at least 20 procedures $[51,52]$. Two other retrospective series have shown that operator experience affects stenting outcome. The first reported significantly higher technical and clinical success rates when the stent was inserted by an operator who had performed at least 10 colonic stent procedures [22]. The second showed a significantly increased immediate perforation rate when colonic stent placement was performed by endoscopists inexperienced in pancreaticobiliary endoscopy [21]. The authors of the latter article explained the lower immediate perforation rate by the skills that therapeutic endoscopic retrograde cholangiopancreatography (ERCP) endoscopists have in traversing complex strictures, understanding fluoroscopy, and deploying stents [21]. Based on the current data, it is difficult to recommend a specific minimum number of performed stent placements. As experience is a significant predictor of success, colonic stent placements should be performed or directly supervised by a competent interventional endoscopist.

\section{Technical considerations of colonic stenting}

\section{RECOMMENDATION}

ESGE suggests that colonic stenting should be performed with the combined use of endoscopy and fluoroscopy. Weak recommendation, low quality evidence.

Colonic stenting can be performed using either the throughthe-scope (TTS) or the over-the-[guide]wire (OTW) technique. The OTW technique is performed using fluoroscopic guidance with or without tandem endoscopic monitoring. Purely radiologic stent placement is performed by advancing the stent deployment system over a stiff guidewire, and technical and clinical success rates of $83 \%-100 \%$ and $77 \%-100 \%$ have been reported in observational studies [55-61]. Retrospective studies that compared endoscopy combined with fluoroscopic guidance versus solely radiography for stent placement show comparable success rates, although some studies show a trend towards higher technical success when either the endoscopic or the combined technique is used compared to solely radiography $[22,48,62-67]$.

\section{RECOMMENDATION}

ESGE recommends not to perform stricture dilation in the setting of colonic stenting.

Strong recommendation, low quality evidence.

Although based on low quality evidence, there are strong indications that stricture dilation either just before or after colonic stent placement adversely affects the clinical outcome and particularly increases the risk of colonic perforation $[13,18,21$, $67,68]$. Pooled analyses of mainly retrospective data from series that mostly included patients with malignant strictures, also revealed an increased risk of perforation after stricture dilation $[63,69,70]$. In addition, no significant effects of balloon dilation on technical success $[48,67]$ and clinical success [67] were observed.

\section{RECOMMENDATION}

ESGE recommends the use of uncovered SEMS in the curative setting.

Strong recommendation, low quality evidence. 


\section{RECOMMENDATION}

ESGE suggests the use of uncovered SEMS in the palliative setting.

Weak recommendation, low quality evidence.

\section{RECOMMENDATION}

ESGE suggests to individually tailor the length of the stent to the length of the stenosis and location of the tumor, whereby the stent should preferably extend beyond the stricture at both ends by $1.5-2 \mathrm{~cm}$.

Weak recommendation, low quality evidence.

A meta-analysis from 2019, including one RCT, seven prospective observational studies, and two retrospective studies, compared covered and uncovered SEMSs either as a bridge to surgery in the curative setting or as palliative treatment. Uncovered SEMSs were associated with fewer complications (risk ratio [RR] 0.57), including less tumor overgrowth (RR 0.29 ) and SEMS migration (RR 0.29), longer SEMS patency (mean duration 18 months), and fewer re-insertions (RR 0.38), although the risk of tumor ingrowth was higher (RR 4.53) [71]. Technical and clinical success did not differ. These observations confirm the results of two earlier meta-analyses, showing less migration but more tumor ingrowth for uncovered SEMS [72, 73]. In the palliative setting, migration can be treated with stent replacement or stent-in-stent techniques [74, 75]. However, in bridge-to-surgery patients, most patients with stent migration are treated with earlier surgery.

Evidence is too limited to recommend on the ideal stent diameter. Comparisons of SEMSs with several diameters did not show any differences in technical success, clinical success, or adverse events including perforation [22,76-79]. Smallercaliber stents were considered to produce less mechanical stress, with a potentially decreased perforation rate [76, 79]. However, a few studies have suggested an association between small-diameter stents $(<24 \mathrm{~mm})$ and adverse events, in particular stent migration [21, 80-82].

Conflicting results regarding ideal stent length have been reported $[13,17,22,48,60,76,83-86]$. Longer stents may allow for better conformability to tumor stricture, especially when located in flexures. Distal stent markers should be located proximal to the obstruction in anticipation of stent foreshortening. It is recommended to use a stent that is long enough to bridge the stenosis and to extend at least $1.5-2 \mathrm{~cm}$ on each side of the lesion, taking into account the degree of shortening after stent deployment.

Several studies have shown no differences in efficacy and safety between different stent designs [58, 75, 87-89].

\section{Clinical indication: colonic stenting as a bridge to elective surgery}

\section{RECOMMENDATION}

ESGE recommends stenting as a bridge to surgery to be discussed, within a shared decision-making process, as a treatment option in patients with potentially curable leftsided obstructing colon cancer as an alternative to emergency resection. This discussion should include the following factors: availability of required stenting expertise, risk of stent-related perforation, higher recurrence rates, similar overall survival and postoperative mortality, lower overall complication rates and permanent stoma rates, higher proportion of laparoscopic one-stage surgery procedures, and technical and clinical failure rates of stenting.

Strong recommendation, high quality evidence.

Interpretation of the literature on stenting as a bridge to surgery can be challenging. There are a large number of retrospective and cohort studies with conflicting results, some of which have been included in meta-analyses. Most randomized trials were published almost a decade ago and were relatively small [41,42,90-95]. The (long-term) results of the two largest and recently completed trials (CREST and ESCO) are becoming available $[94,96]$.

Details of the patient populations often lack clear definitions and there are heterogeneities of interventions and study populations. These include stage and curability of the patients' disease, severity of obstruction based on both clinical symptoms and imaging findings, and type of emergency surgery performed. Concerning the latter issue, creation of only a decompressing stoma in the emergency setting is also a bridging technique similar to colonic stenting, and it has a different risk profile compared with emergency resection. Treatment decisions are highly dependent on treatment intent, but many studies on stenting as bridge to surgery mix palliative and curative cases. Furthermore, the intention of treatment is often difficult to determine in the emergency setting, and the effectiveness of multimodality treatment in stage IV disease is improving.

Most of the literature concerns left-sided obstructing colon cancer excluding (distal) rectal cancers, but similar clinical issues have been raised regarding right-sided malignant obstruction proximal to the splenic flexure [97-99].

Sensitivity analysis has shown that experience and volume might influence long-term outcome, based on meta-analyses of studies with technical success rates $<90 \%$ versus $\geq 90 \%$, and studies including $<40$ versus $\geq 40$ SEMS cases [100]. Therefore, unit experience and expertise may also influence treatment decisions.

The literature shows technical and clinical failure rates of up to $25 \%$ for colonic stenting, influenced by expertise, technique, and location of the obstruction (i.e., colonic/sigmoid flexures) 
[76, 101 - 106]. Considering short-term outcomes, meta-analyses have shown lower morbidity rates after stenting as a bridge to surgery than emergency surgery $[107,108]$, similar postoperative mortality rates [107], and a higher proportion of primary anastomoses [108].

There are few studies available on the oncological implications of stent-related perforation. These reports mostly consider clinically overt perforations. In a subgroup analysis of the Dutch Stent-in 2 trial, Sloothaak et al. demonstrated an increased recurrence risk in patients with stent-related perforation (clinically overt and occult, $n=6$ ) compared to patients without a stent-related perforation [109]. Similar comparisons of patients with versus without a stent-related perforation were performed in a Dutch population-based study (17 perforations) showing, respectively, a 3-year locoregional recurrence of $18 \%$ versus $11 \%(P=0.43)$, 3-year disease-free survival of $49 \%$ versus $60 \%(P=0.72)$, and 3 -year overall survival of $61 \%$ and $75 \%(P=$ $0.53)$ [110]. Currently available data are still underpowered but suggest a negative impact of stent-related perforation on oncological outcomes. The initial RCTs showed relatively high rates of bowel perforation, which led to premature termination of two trials [41,42]. More recent trials have shown perforation rates below $10 \%$ [94]. Sensitivity analyses revealed that 3-year overall survival was significantly better in studies with a perforation rate less than $8 \%$ compared to those with $8 \%$ or higher [100]. It has been argued that oncologic outcome after perforations might be different depending on the cause of the perforation or the presence of symptoms (e. g. guidewire- or stent expansion-related, clinically silent or overt), but too few data are available to confirm this. Other similar factors, such as forceful stent expansion of the tumor, could introduce cancer cells into vessels, thereby facilitating dissemination [111]. Furthermore, colonic stenting may promote perineural invasion as detected in resected specimens, albeit not translating into poorer oncologic outcomes [112,113]. So far, there are insufficient data to support these theories and findings.

A recent meta-analysis showed higher overall recurrence (37.0\% vs. $25.9 \%$; RR 1.425, $95 \%$ confidence interval $[\mathrm{Cl}]$ $1.002-2.028 ; P=0.049)$ and systemic recurrence (RR 1.627, $95 \% \mathrm{Cl} 1.009-2.621, P=0.046)$ in the colonic stenting group. Nevertheless, this did not translate into significantly worse 3year disease-free survival or 3-year overall survival [107]. These results were confirmed by another meta-analysis that showed no differences in 5-year disease-free survival and 5-year overall survival [100]. Moreover, unpublished data related to the ESCO study and the CREST study (A. Arezzo and J. Hill, Guideline discussion, Amsterdam, 3 September 2019) show similar overall, systemic, and local recurrences for both groups.

Long-term advantages of colonic stenting over emergency resection include a lower permanent stoma risk and higher primary anastomosis rates $[100,110]$. Considering the outcome measures of hospital stay and quality of life, the data are sparse and inconclusive.

For individual patients, decision making might be influenced by the relative importance of particular end points. For a young fit patient, the chance of stoma reversal in the long run is likely to be high [110], while a potentially higher risk of distant recur- rence might result in a preference for emergency resection. For elderly patients, short-term outcomes might be more important, especially the lower risk of complications and lower chance of a stoma.

As an alternative to colonic decompression, insertion of a transanal decompression tube (TDT) is only rarely done, with most case series being performed in south-east Asian countries. A recent meta-analysis on TDT versus colonic stenting as a bridge to surgery reported lower technical success, lower clinical success, fewer primary tumor resections, fewer primary anastomoses, and more stomas for TDT than colonic stenting [114]. Therefore, ESGE does not recommend TDT placement over colonic stent placement.

- Table 1 summarizes the high quality evidence from metaanalyses regarding the short-term outcomes of SEMS placement as a bridge to surgery versus emergency surgery, and - Table 2 summarizes the oncological outcomes.

\section{RECOMMENDATION}

ESGE suggests reluctance regarding colonic stenting of long-segment stenosis in a curative setting.

Weak recommendation, low quality evidence.

A post hoc evaluation of a prospective observational study revealed that stricture length of at least $5 \mathrm{~cm}$ was associated with technical difficulty in colonic stenting [48], whereas another retrospective study comparing strictures up to $4 \mathrm{~cm}$ and longer than $4 \mathrm{~cm}$ revealed no significant differences in technical success, clinical success, and re-obstruction [122]. Boyle et al. showed that shorter strictures and wider angulation distal to the obstruction were significantly associated with successful deployment and clinical decompression [123]. Furthermore, perforation was associated with longer strictures. In addition, malignant strictures had a shorter median length compared to strictures due to diverticular disease or external compression (40 vs. $65 \mathrm{~mm}, P<0.001$ ). Notably, a meta-analysis showed that perforation rates were significantly higher for benign than for malignant strictures (18.4\% versus $7.5 \%$ ) [70].

Based on these findings, care should be taken when stenting a relatively long stricture as this might be caused by a benign lesion (e.g. diverticulitis), with a potentially higher risk of perforation. Furthermore, locally advanced tumors (cT4] might have an indication for induction therapy, which might be another argument for refraining from colonic stenting in large bulky lesions.

\section{RECOMMENDATION}

ESGE suggests a time interval of approximately 2 weeks until resection when colonic stenting is performed as bridge to elective surgery in patients with curable leftsided colon cancer.

Weak recommendation, low quality evidence. 
- Table 1 Meta-analyses of short-term outcomes of self-expandable metal stent (SEMS) placement as a bridge to elective surgery in patients with malignant colonic obstruction.

\begin{tabular}{|c|c|c|c|}
\hline $\begin{array}{l}\text { First author, } \\
\text { year }\end{array}$ & Study population & Results & $\begin{array}{l}\text { Study design } \\
\text { Level of evidence }\end{array}$ \\
\hline $\begin{array}{l}\text { Foo, } 2019 \\
{[107]}\end{array}$ & $\begin{array}{l}\text { Patients with acute left-sided } \\
\text { malignant colonic obstruction } \\
7 \text { RCTs } \\
\text { Preoperative SEMS }(n=222) \\
\text { Emergency surgery }(n=226)\end{array}$ & $\begin{array}{l}\text { SEMS vs. emergency surgery: } \\
\text { " Lower overall complication risk (RR } 0.605,95 \% \mathrm{Cl} 0.382-0.958 \text { ) } \\
\text { " No significant difference in 30-day mortality (RR } 0.963,95 \% \mathrm{Cl} \\
0.468-1.982 \text { ) }\end{array}$ & $\begin{array}{l}\text { Meta-analysis of } \\
\text { RCTs } \\
\text { High quality } \\
\text { evidence }\end{array}$ \\
\hline $\begin{array}{l}\text { Yang, } 2018 \\
{[108]}\end{array}$ & $\begin{array}{l}\text { Patients with acute left-sided } \\
\text { obstructive colorectal cancer } \\
8 \text { RCTs } \\
\text { Preoperative SEMS }(n=251) \\
\text { Emergency surgery }(n=246)\end{array}$ & $\begin{array}{l}\text { SEMS vs. emergency surgery: } \\
\text { - Lower direct stoma rate (OR } 0.46,95 \% \mathrm{Cl} 0.30-0.70) \\
\text { - Higher successful primary anastomosis rate (OR } 2.29,95 \% \mathrm{Cl} 1.52 \text { - } \\
3.45 \text { ) } \\
\text { - Fewer post-procedural complications (OR } 0.39,95 \% \mathrm{Cl} 0.18-0.82 \text { ) } \\
\text { - Fewer wound infections (OR } 0.49,95 \% \mathrm{Cl} 0.27-0.87 \text { ) }\end{array}$ & $\begin{array}{l}\text { Meta-analysis of } \\
\text { RCTs } \\
\text { High quality } \\
\text { evidence }\end{array}$ \\
\hline $\begin{array}{l}\text { Allievi, } 2017 \\
\text { [115] }\end{array}$ & $\begin{array}{l}\text { Patients with left-sided malig- } \\
\text { nant colorectal obstruction } \\
7 \text { RCTs } \\
\text { Preoperative SEMS }(n=222) \\
\text { Emergency surgery }(n=226)\end{array}$ & $\begin{array}{l}\text { SEMS vs. emergency surgery: } \\
\text { - Fewer postoperative complications (RR 0.6, } 95 \% \mathrm{Cl} 0.38-0.96) \\
\text { - Fewer stomas (RR } 0.64,95 \% \mathrm{Cl} 0.51-0.80 \text { ) } \\
\text { - No significant difference in primary anastomosis rate (RR } 1.20,95 \% \mathrm{Cl} \\
0.95-1.52 \text { ) } \\
\text { - No significant difference in anastomotic leakages (RR } 0.93,95 \% \mathrm{Cl} \\
\quad 0.45-1.92 \text { ) } \\
\text { - No significant difference in in-hospital mortality (RR } 0.98,95 \% \mathrm{Cl} \\
0.53-1.82 \text { ) }\end{array}$ & $\begin{array}{l}\text { Meta-analysis of } \\
\text { RCTs } \\
\text { High quality } \\
\text { evidence }\end{array}$ \\
\hline $\begin{array}{l}\text { Arezzo, } 2017 \\
{[116]}\end{array}$ & $\begin{array}{l}\text { Patients with left-sided malig- } \\
\text { nant colonic obstruction } \\
8 \text { RCTs } \\
\text { Preoperative SEMS }(n=251) \\
\text { Emergency surgery }(n=246)\end{array}$ & $\begin{array}{l}\text { SEMS vs. emergency surgery: } \\
\text { - Lower overall morbidity rate (RR } 0.59,95 \% \mathrm{Cl} 0.38-0.93) \\
\text { - Fewer temporary stomas (RR } 0.67,95 \% \mathrm{Cl} 0.54-0.83) \\
\text { " Higher primary anastomosis rate (RR } 1.29,95 \% \mathrm{Cl} 1.01-1.66) \\
\text { - No significant difference in overall mortality< } 60 \text { days after } \\
\text { surgery (RR } 0.98,95 \% \mathrm{Cl} 0.53-1.82)\end{array}$ & $\begin{array}{l}\text { Meta-analysis of } \\
\text { RCTs } \\
\text { High quality } \\
\text { evidence }\end{array}$ \\
\hline $\begin{array}{l}\text { Wang, } 2017 \\
{[117]}\end{array}$ & $\begin{array}{l}\text { Patients with left-sided } \\
\text { colorectal cancer with } \\
\text { malignant obstruction } \\
9 \text { RCTs } \\
\text { Preoperative SEMS }(n=281) \\
\text { Emergency surgery }(n=313)\end{array}$ & $\begin{array}{l}\text { SEMS vs. emergency surgery: } \\
\text { " Higher one-stage anastomosis rate (OR } 2.56,95 \% \mathrm{Cl} 1.79-3.66 \text {, } \\
P<0.0001) \\
\text { " No significant difference in anastomotic leakages (OR } 1.12,95 \% \mathrm{Cl} \\
\quad 0.55-2.30, P=0.75) \\
\text { - Lower postoperative mortality rate (OR } 0.51,95 \% \mathrm{Cl} 0.26-0.98 \text {, } \\
P=0.04 \text { ) } \\
\text { - Fewer minor complications (OR } 0.65,95 \% \mathrm{Cl} 0.45-0.93, P=0.02 \text { ) }\end{array}$ & $\begin{array}{l}\text { Meta-analysis of } \\
\text { RCTs } \\
\text { High quality } \\
\text { evidence }\end{array}$ \\
\hline $\begin{array}{l}\text { Huang, } 2014 \\
\text { [118] }\end{array}$ & $\begin{array}{l}\text { Patients with acute left-sided } \\
\text { malignant colonic obstruction } \\
7 \text { RCTs } \\
\text { Preoperative SEMS }(n=195) \\
\text { Emergency surgery }(n=187)\end{array}$ & $\begin{array}{l}\text { SEMS vs. emergency surgery: } \\
\text { " Higher primary anastomosis rate (OR } 2.01,95 \% \mathrm{Cl} 1.21-3.31) \\
\text { - Lower overall complication rate (OR } 0.30,95 \% \mathrm{Cl} 0.11-0.86) \\
\text { - Fewer wound infections (OR } 0.31,95 \% \mathrm{Cl} 0.14-0.68) \\
\text { - No significant difference in anastomotic leakage rate (OR } 0.74,95 \% \mathrm{Cl} \\
\\
0.33-1.67) \\
\text { - No significant difference in mortality (OR } 0.88,95 \% \mathrm{Cl} 0.40-1.96)\end{array}$ & $\begin{array}{l}\text { Meta-analysis of } \\
\text { RCTs } \\
\text { High quality } \\
\text { evidence }\end{array}$ \\
\hline $\begin{array}{l}\text { Zhao, } 2014 \\
\text { [119] }\end{array}$ & $\begin{array}{l}\text { Patients with left-sided malig- } \\
\text { nant colonic obstruction } \\
6 \text { RCTs } \\
\text { Preoperative SEMS }(n=136) \\
\text { Emergency surgery }(n=137)\end{array}$ & 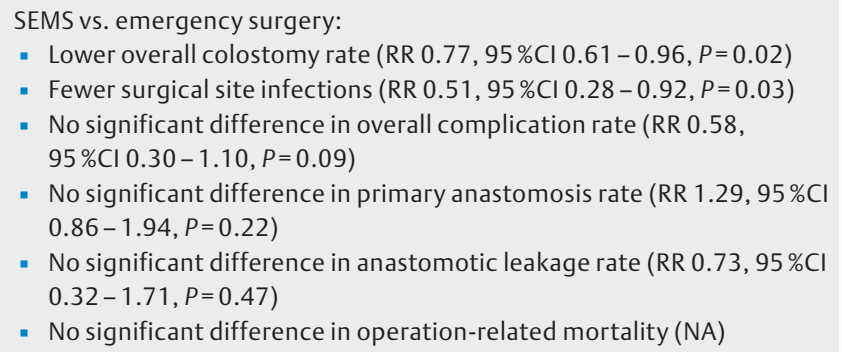 & $\begin{array}{l}\text { Meta-analysis of } \\
\text { RCTs } \\
\text { High quality } \\
\text { evidence }\end{array}$ \\
\hline
\end{tabular}


- Table 2 Oncological outcome of self-expandable metal stent (SEMS) placement versus emergency surgery in patients with malignant colonic obstruction.

\begin{tabular}{|c|c|c|c|}
\hline $\begin{array}{l}\text { First author, } \\
\text { year }\end{array}$ & Study population & Results & $\begin{array}{l}\text { Study design } \\
\text { Level of evidence }\end{array}$ \\
\hline $\begin{array}{l}\text { Foo, } \\
2019 \text { [107] }\end{array}$ & $\begin{array}{l}\text { Patients with acute left-sided } \\
\text { malignant colonic obstruction } \\
7 \text { RCTs } \\
\text { Preoperative SEMS }(n=222) \\
\text { Emergency surgery }(n=226)\end{array}$ & $\begin{array}{l}\text { SEMS vs. emergency surgery: } \\
\text { - Higher overall recurrence rate (RR } 1.425,95 \% \mathrm{Cl} 1.002-2.028) \\
\text { - Higher systemic recurrence rate (RR } 1.627,95 \% \mathrm{Cl} 1.009-2.621 \text { ) } \\
\text { - No significant difference in locoregional recurrence (RR } 1.110 \text {, } \\
95 \% \mathrm{Cl} 0.593-2.078 \text { ) } \\
\text { - No significant difference in 3-year disease-free survival (OR } 1.429 \text {, } \\
95 \% \mathrm{Cl} 0.801 \text { - } 2.550 \text { ) } \\
\text { - No significant difference in 3-year overall survival (OR } 1.659 \text {, } \\
95 \% \mathrm{Cl} 0.930 \text { - } 2.962 \text { ) }\end{array}$ & $\begin{array}{l}\text { Meta-analysis of } \\
\text { RCTs } \\
\text { High quality } \\
\text { evidence }\end{array}$ \\
\hline $\begin{array}{l}\text { Amelung, } \\
2018 \text { [100] }\end{array}$ & $\begin{array}{l}\text { Patients with acute left-sided } \\
\text { malignant colonic obstruction } \\
5 \text { RCTs, } 4 \text { prospective nonrando- } \\
\text { mized comparative studies, } 12 \\
\text { retrospective comparative } \\
\text { studies } \\
\text { Preoperative SEMS }(n=938) \\
\text { Emergency surgery }(n=981)\end{array}$ & $\begin{array}{l}\text { SEMS vs. emergency surgery: } \\
\text { - No significant difference in locoregional recurrence (OR } 1.32 \text {, } \\
95 \% \mathrm{Cl} 0.78-2.23 \text { ) } \\
\text { - No significant difference in overall recurrence (OR 1.06, } 95 \% \mathrm{Cl} \\
0.76-1.47 \text { ) } \\
\text { - No significant difference in 3-year disease-free survival (OR } 0.96 \text {, } \\
95 \% \mathrm{Cl} 0.73-1.26 \text { ) and } 5 \text {-year disease-free survival (OR } 0.86 \text {, } \\
95 \% \mathrm{Cl} 0.54-1.36 \text { ) } \\
\text { - No significant difference in 3-year overall survival (OR } 0.85,95 \% \mathrm{Cl} \\
0.68-1.08 \text { ) and 5-year overall survival (OR } 1.04,95 \% \mathrm{Cl} 0.68-1.57 \text { ) }\end{array}$ & $\begin{array}{l}\text { Meta-analysis } \\
\text { Moderate quality } \\
\text { evidence }\end{array}$ \\
\hline $\begin{array}{l}\text { Yang, } \\
2018 \text { [108] }\end{array}$ & $\begin{array}{l}\text { Patients with acute left-sided } \\
\text { obstructive colorectal cancer } \\
8 \text { RCTs } \\
\text { Preoperative SEMS }(n=251) \\
\text { Emergency surgery }(n=246)\end{array}$ & $\begin{array}{l}\text { SEMS vs. emergency surgery: } \\
\text { - Higher odds of tumor recurrence (OR 1.79, } 95 \% \mathrm{Cl} 1.09-2.93)\end{array}$ & $\begin{array}{l}\text { Meta-analysis of } \\
\text { RCTs } \\
\text { High quality } \\
\text { evidence }\end{array}$ \\
\hline $\begin{array}{l}\text { Arezzo, } \\
2017[116]\end{array}$ & $\begin{array}{l}\text { Patients with left-sided malig- } \\
\text { nant colonic obstruction } \\
8 \text { RCTs } \\
\text { Preoperative SEMS }(n=251) \\
\text { Emergency surgery }(n=246)\end{array}$ & $\begin{array}{l}\text { SEMS vs. emergency surgery: } \\
\text { - No significant difference in relative risk of tumor recurrence } \\
\text { (RR } 1.80,95 \% \mathrm{Cl} 0.91-3.54)\end{array}$ & $\begin{array}{l}\text { Meta-analysis of } \\
\text { RCTs } \\
\text { High quality evi- } \\
\text { dence }\end{array}$ \\
\hline $\begin{array}{l}\text { Arezzo, } \\
2017 \text { [94] }\end{array}$ & $\begin{array}{l}\text { Patients with acute symptomatic } \\
\text { malignant left-sided large-bowel } \\
\text { obstruction (splenic flexure to } 15 \\
\text { cm from anal margin as diag- } \\
\text { nosed by CT imaging) }(n=115) \\
\text { - SEMS as bridge to surgery } \\
\quad(n=56) \\
\text { - Emergency surgery }(n=59)\end{array}$ & $\begin{array}{l}\text { Recurrence at median follow-up of } 36 \text { months }(P=0.685) \\
\text { - SEMS } 30.3 \% \\
\text { - Surgery } 33.9 \% \\
\text { No significant difference in overall survival }(P=0.998) \text { and } \\
\text { progression-free survival }(P=0.893)\end{array}$ & $\begin{array}{l}\text { RCT } \\
\text { High quality } \\
\text { evidence }\end{array}$ \\
\hline $\begin{array}{l}\text { Ceresoli, } \\
2017 \text { [120] }\end{array}$ & $\begin{array}{l}\text { Patients with malignant left-sided } \\
\text { colonic obstruction } \\
5 \text { RCTs, } 3 \text { prospective nonrando- } \\
\text { mized comparative studies, } 9 \\
\text { retrospective comparative studies } \\
\text { Preoperative SEMS }(n=688) \\
\text { Emergency surgery }(n=655)\end{array}$ & $\begin{array}{l}\text { SEMS vs. emergency surgery: } \\
\text { - No significant difference in overall recurrence (RR } 1.11,95 \% \mathrm{Cl} \\
0.84-1.47, P=0.47 \text { ) } \\
\text { - No significant difference in local recurrence (RR } 1.41,95 \% \mathrm{Cl} \\
0.89-2.23, P=0.14 \text { ) } \\
\text { - No significant difference in 3-year recurrence (RR } 1.15,95 \% \mathrm{Cl} \\
0.95-1.39, P=0.14 \text { ) } \\
\text { - No significant difference in } 5 \text {-year recurrence (RR } 1.05,95 \% \mathrm{Cl} \\
0.88-1.25, P=0.59 \text { ) } \\
\text { - No significant difference in 3-year mortality (RR } 0.90,95 \% \mathrm{Cl} \\
0.73-1.12, P=0.34 \text { ) } \\
\text { - No significant difference in } 5 \text {-year mortality (RR } 1.00,95 \% \mathrm{Cl} \\
0.82-1.22, P=0.99 \text { ) }\end{array}$ & $\begin{array}{l}\text { Meta-analysis } \\
\text { Moderate quality } \\
\text { evidence }\end{array}$ \\
\hline $\begin{array}{l}\text { Matsuda, } \\
2015 \text { [121] }\end{array}$ & $\begin{array}{l}\text { Patients with malignant large- } \\
\text { bowel obstruction } \\
11 \text { studies of which } 2 \text { RCTs, } 2 \text { pro- } \\
\text { spective nonrandomized com- } \\
\text { parative studies, } 7 \text { retrospective } \\
\text { comparative studies } \\
\text { Preoperative SEMS ( } n=432) \\
\text { Emergency surgery }(n=704)\end{array}$ & $\begin{array}{l}\text { SEMS vs. emergency surgery: } \\
\text { - No significant difference in overall survival (RR } 0.95,95 \% \mathrm{Cl} \\
0.75-1.21, P=0.66 \text { ) } \\
\text { - No significant difference in disease-free survival (RR } 1.06,95 \% \mathrm{Cl} \\
0.91-1.24, P=0.43 \text { ) } \\
\text { - No significant difference in recurrence (RR } 1.13,95 \% \mathrm{Cl} 0.82-1.54 \text {, } \\
P=0.46 \text { ) }\end{array}$ & $\begin{array}{l}\text { Meta-analysis } \\
\text { Moderate quality } \\
\text { evidence }\end{array}$ \\
\hline
\end{tabular}


- Table 2 (Continuation)

\begin{tabular}{|c|c|c|c|}
\hline $\begin{array}{l}\text { First author, } \\
\text { year }\end{array}$ & Study population & Results & $\begin{array}{l}\text { Study design } \\
\text { Level of evidence }\end{array}$ \\
\hline $\begin{array}{l}\text { Sloothaak, } \\
2014 \text { [109] }\end{array}$ & $\begin{array}{l}\text { Patients with acute left-sided } \\
\text { malignant colonic obstruction } \\
(n=98) \\
\text { SEMS as bridge to surgery }(n=47) \\
\text { Emergency surgery }(n=51)\end{array}$ & $\begin{array}{l}\text { SEMS vs. emergency surgery: } \\
\text { - Median (range) follow-up: } 41(19-55) \text { vs. } 45(35-60) \text { months } \\
\text { - Higher 5-year overall recurrence rate }(P=0.027) \\
\text { " No significant difference in locoregional recurrence rate }(P=0.052) \\
\text { Patients with a stent perforation: } n=6 \\
\text { Cumulative incidence of overall recurrences }(P<0.01) \text { : } \\
\text { - Patients with stent perforation: } 83 \%(95 \% \mathrm{Cl} 58 \%-100 \%) \\
\text { - Nonperforated stent patients: } 34 \%(95 \% \mathrm{Cl} 18 \%-65 \%) \\
\text { - Emergency surgery: } 26 \%(95 \% \mathrm{Cl} 14 \%-47 \%) \\
\text { 5-year cumulative incidence of locoregional recurrences }(P=0.053) \text { : } \\
\text { - Patients with stent perforation: } 50 \%(95 \% \mathrm{Cl} 22 \%-100 \%) \\
\text { - Nonperforated stent patients: } 10 \%(95 \% \mathrm{Cl} 3 \%-41 \%)\end{array}$ & $\begin{array}{l}\text { Follow-up data of } \\
\text { RCT [31] } \\
\text { Low quality } \\
\text { evidence }\end{array}$ \\
\hline
\end{tabular}

The time interval for surgery after colonic stenting has to be discussed and analyzed depending on the balance between stent-related adverse events (reduced by a short interval) and surgical outcomes (improved by a longer delay). No prospective comparative data are available on the impact of this period (short vs. long) for the surgery, complications, and overall or disease-free survival.

In a meta-analysis of RCTs that compared colonic stenting as a bridge to surgery versus emergency surgery, the time intervals in the colonic stenting arms ranged from 3 days to 4 weeks [107]. In recently published, large prospective studies, most of the stent-related complications occurred within 7 days [105, 124]. Conflicting data are noted in retrospective studies regarding the association between this interval and postoperative complications or disease-free recurrence and survival [78, 125 - 130]. The risk of anastomotic leakage was significantly reduced when surgery was delayed for 10 days or longer in a retrospective analysis [130]. In another study, a cutoff value of 15 days was proposed to significantly reduce the risk of postoperative complications (OR for an interval $\leq 15$ days, $13.0,95 \% \mathrm{Cl}$ $1.0-167.0$, area under the curve 0.793) [78]. Considering risk of recurrence, one study reported that a period $\geq 18$ days was shown to be an independent risk factor (OR 5.1, 95\%Cl 1.6 $15.8, P=0.005)$ [129]. Nevertheless, other studies did not find any significant impact of time interval on outcome [127, 128].

Based on these data and the clinical experience of experts, it seems that a certain period of waiting after colonic stenting might be beneficial in order to optimize the patient's clinical condition, and thereby reduce the risks of subsequent surgical resection. Since stent perforation often occurs very early, it seems that reducing the interval to resection would not prevent this complication. In the absence of good quality evidence, the time interval before surgery should be dictated by optimization of nutritional status and adequate management of comorbidities; this may require a few weeks.

There is a tendency towards induction chemotherapy in locally advanced colon cancer. The FOXTROT trial has recently been presented at an American Society of Clinical Oncology (ASCO) meeting [131], and showed a significant decrease in
R1 resection rate and a nonsignificant trend towards better oncological outcome at 2 years. However, only a few patients in this trial underwent colonic stenting as a bridge to surgery, and separate data are not (yet) available. Other studies of chemotherapy during the bridging interval in the curative setting were not identified. Therefore, no recommendation could be formulated.

\section{RECOMMENDATION}

ESGE suggests that a decompressing stoma as bridge to elective surgery is a valid option if the patient is not a candidate for colonic stenting or when stenting expertise is not available.

Weak recommendation, low quality evidence.

Four retrospective studies have been published regarding the role of a decompressing stoma as bridge to elective surgery compared to colonic stenting for left-sided colon cancer [102, 132 -134]. In two of these studies, propensity scores were used to correct for baseline differences $[102,132]$.

Three of the four studies report a larger total number of interventions for decompressing stoma construction than for colonic stenting [132-134]. In addition, patients with decompressing stoma had more primary anastomoses constructed $[102,132]$ and had more stomas in situ after resection [132, 134]. Conflicting results on morbidity have been published. Lower major morbidity rates have been reported for decompressing stoma with no significant differences in total complication rate $[132,134]$, although others reported higher complication rates for decompressing stoma [102]. In addition, the permanent stoma rate did not differ between the two bridge-tosurgery techniques $[102,132,134]$. Locoregional recurrence [132], disease-free survival $[102,132,134]$, and overall survival $[132,134]$ were similar. However, in one study, decompressing stoma showed better overall survival than colonic stenting, suggesting an important role for noncancer-related deaths [102]. 
Based on the currently available literature, construction of a stoma seems a valid alternative for decompressing the colon as a bridge to surgery. This is especially relevant in circumstances where sufficient experience with colonic stenting is not available, the patient does not seem to be a good candidate for colonic stenting (e.g. long stenosis or locally advanced disease that requires induction therapy), or if colonic stent placement technically failed. A decompressing stoma also allows patients to recover with a higher chance of a primary anastomosis [102, 132]. The disadvantage is that all patients will have a stoma for a certain period of time, which often requires a third intervention to restore continuity. The lack of good quality data on these two interventions suggests the need for a randomized trial.

\section{Malignant obstruction of the proximal colon}

\section{RECOMMENDATION}

ESGE suggests consideration of colonic stenting for malignant obstruction of the proximal colon either as a bridge to surgery or in a palliative setting.

Weak recommendation, low quality evidence.

Several retrospective series have shown that colonic stenting may be successful in malignant obstruction of the proximal colon (MOPC) (i.e., proximal to the splenic flexure) $[13,22$, 135 -138]. Studies comparing the technical success rates between stent placement in right-sided and left-sided colon cancers show conflicting results with a tendency to lower technical success rates in the right-sided colon $[13,22,53,122,135$, 139]. A post-hoc analysis of a prospective multicenter study demonstrated longer procedure times for stents placed in the right-sided colon [48], with other studies showing similar clinical success, adverse event, and re-intervention rates, as well as similar 5-year overall and disease-free survival when compared to the left-sided colon $[13,22,97,99,135-138,140]$.

Comparing with emergency surgery, a recent systematic review showed lower mortality ( $0 \%$ vs. $10.8 \%, P=0.009$ ), less major morbidity $(0.8 \%$ vs $23.9 \%, P=0.049)$, and lower risk of anastomotic leakage (0\% vs. 9.1\%) for colonic stenting in patients with MOPC [99].

Regarding the palliative setting, two retrospective series including both palliative and curative patients presented conflicting data $[98,141]$. The first study showed fewer early complications ( $7.2 \%$ versus $30.5 \%, P=0.003)$ and shorter hospital stay ( 3.5 vs. 8 days, $P<0.001$ ], but lower clinical success $(78 \%$ vs. $100 \%, P<0.001)$ and lower patency $(73.9 \%$ vs. $94.4 \%, P=$ 0.02 ) for colonic stenting compared to emergency surgery in MOPC [141]. The second study was not able to find any differences between matched colonic stenting and primary surgery patients regarding morbidity, mortality, or hospital stay, although temporary stoma rate was lower in the stent group ( $0 \%$ vs. $21.1 \%, P=0.04$ ) [98]. In general, based on low quality evidence, a trend towards lower morbidity and mortality exists for MOPC patients treated with colonic stenting compared to emergency surgery. However, it should be mentioned that stenting of the right-sided colon might be challenging and probably requires more experience.

\section{Clinical indication: palliative colonic stenting}

RECOMMENDATION

ESGE recommends colonic stenting as the preferred treatment for palliation of malignant colonic obstruction. Strong recommendation, high quality evidence.

Four systematic reviews and/or meta-analyses, including randomized and nonrandomized comparative studies, have compared colonic stenting and surgery for palliation of malignant colonic obstruction [142-145]. The technical success of stent placement ranged from $88 \%$ to $100 \%$, while the initial clinical relief of obstruction was significantly higher after palliative surgery compared to colonic stenting ( $96 \%$ vs. $86.1 \%, P=$ 0.02 ) [143]. Conflicting results have been reported regarding short-term mortality, with lower 30-day mortality for colonic stenting in two meta-analyses $[144,145]$ and no significant differences found in the other studies $[142,143]$. No significant differences in overall morbidity were found between the stent group and the surgery group $[143,145]$, although two metaanalyses revealed more short-term complications in the palliative surgery group, while late complications were more frequent in the stent group $[144,145]$. No significant differences in morbidity were found when comparing colonic stenting and decompressing stoma in the palliative setting [146].

Placement of a colonic stent was significantly associated with shorter hospitalization and a lower intensive care unit admission rate $[142,143,145,147]$, while permitting a shorter time to initiation of chemotherapy $[145,148]$. Stenting also resulted in shorter hospital stay when compared to decompressing stoma in the palliative setting (OR 0.50, 95\% Cl 0.26-0.97, $P=0.04$ ) [146]. Surgical stoma formation was significantly lower after palliative colonic stenting compared with emergency surgery [143-145].

In an RCT by Young et al. [147], the surgery group had significantly reduced quality of life if compared with the stent group from baseline to 1 and 2 weeks $(P=0.001$ and $P=0.012$, respectively), and from baseline to 12 months $(P=0.01]$. A post hoc analysis of the same RCT revealed lower total costs for stenting than for surgery [149].

A post hoc analysis of a prospective multicenter study and several retrospective studies showed lower technical success $[48,150]$ and an increased complication rate $[151,152]$ for colonic stenting in patients with peritoneal metastases. Another series, that focused on the outcomes of secondary stent insertion after initial stent failure, reported a significantly decreased stent patency in the setting of peritoneal metastases (118 days vs. 361 days) [13]. 


\section{RECOMMENDATION}

ESGE recommends chemotherapy as a safe treatment in patients who have undergone palliative colonic stenting. Strong recommendation, low quality evidence.

\section{RECOMMENDATION}

ESGE suggests that antiangiogenic therapy (e.g. bevacizumab) can be considered in patients following colonic stenting.

Weak recommendation, low quality evidence.

\section{RECOMMENDATION}

ESGE does not suggest colonic stenting while patients are receiving antiangiogenic therapy, such as bevacizumab. Weak recommendation, low quality evidence.

It has been speculated that chemotherapy during colonic stenting might induce stent-related complications, in particular perforation. In a retrospective study of 38 patients evaluating the safety and efficacy of chemotherapy following palliative colonic stenting [153], stenting showed a 30-day complication rate of $2.5 \%$, and the toxicity of antiangiogenic drugs was not enhanced by stent insertion. The risk of perforation was $8 \%$ and occurred from 2 to 15 months after stent insertion.

In a retrospective series of 87 patients who received either chemotherapy without bevacizumab $(n=47)$, or chemotherapy with bevacizumab $(n=10)$, or no chemotherapy $(n=30)$, overall perforation risk was $10 \%$ [154]. The risk of perforation was $13 \%$ for patients who did not receive chemotherapy, $6 \%$ for patients who did receive chemotherapy but no bevacizumab, and $20 \%$ for patients who received chemotherapy and bevacizumab, suggesting a higher perforation risk for patients who received bevacizumab. In a series of 353 patients with stage IV colon cancer, Park et al. found similar perforation rates in stent patients with bevacizumab $(n=96)$ and without bevacizumab ( $n=257)$, namely $7.3 \%$ and $7.0 \%(P=0.93)$, respectively. Moreover, chemotherapy was not a risk factor for complications in patients treated with colonic stenting, and chemotherapy significantly decreased the risk of mortality (hazard ratio [HR] $0.464,95 \% \mathrm{Cl} 0.315-0.683, P<0.001)$ [151].

In a series of 1008 patients who received bevacizumab for metastatic colorectal cancer, the risk of complications necessitating surgery was $5.9 \%$. In patients already receiving bevacizumab, stent insertion was a significant risk factor for complications requiring surgery ( $\mathrm{HR} 5.687,95 \% \mathrm{Cl} 2.372-13.637, P<$ $0.001)$ [155].

\section{RECOMMENDATION}

ESGE suggests consideration of colonic stenting as an alternative to decompressive surgery as palliative treatment for obstruction caused by extracolonic malignancy, although technical and clinical success rates are inferior to those reported in stenting of primary colonic cancer. Weak recommendation, low quality evidence.

Relief of large-bowel obstruction caused by extracolonic malignancy (ECM) by means of colonic stenting has been studied mainly retrospectively. Technical and clinical success rates of stenting for ECM have been reported with ranges $67 \%-96 \%$ and $20 \%-96 \%$, respectively [ $156-161]$. These are considered inferior to those reported for stenting of primary colonic cancer, although conflicting results have been published $[13,17$, $21,81,150,162-164]$. In comparison to emergency decompressive surgery, colonic stenting for ECM has shown significantly fewer complications [158].

Peritoneal metastasis as the cause of ECM has been associated with lower technical and clinical success rates $[67,150,152]$ and more adverse events [67]. However, a few studies revealed higher delayed perforation rates in patients without peritoneal metastases $[77,152]$. In some studies, tumor origin had no impact on technical success $[22,53,86]$, clinical success $[22,86]$, adverse events $[17,22,86]$, early and delayed perforation, nor 30-day mortality after stent placement $[77,123]$. Altogether, palliative stenting of ECM is to be considered in order to avoid decompressing surgery in these patients because of the high risk of postoperative morbidity and mortality $[157,159,160,162]$.

\section{Adverse events related to colonic stenting}

\section{RECOMMENDATION}

In the palliative setting, ESGE recommends endoscopic re-intervention by stent-in-stent placement for colonic stent obstruction, or stent replacement when migration occurs.

Strong recommendation, low quality evidence.

\section{RECOMMENDATION}

In the curative setting, ESGE suggests early surgery rather than repeat colonic stenting when stent obstruction or migration occurs in patients being bridged to surgery. Weak recommendation, low quality evidence. 


\section{RECOMMENDATION}

ESGE recommends that emergency resection should be considered in patients with stent-related perforation. Strong recommendation, low quality evidence.

Colonic stent placement in patients with malignant largebowel obstruction is associated with potential adverse events. Overall complication rates for colonic stenting commonly approach $20 \%-30 \%$ in case series, with higher rates reported in RCTs, although the 30-day stent-related mortality is less than $4 \%$ [144]. The main complications include perforation, stent failure, migration, and re-obstruction. Delayed complications occur in up to $20 \%$ of patients, most commonly stent malfunction or perforation $[17,65,76,103,105,145,165-176]$. For purely palliative indications, a meta-analysis of 410 patients reported short-term and long-term complication rates of 26.2 $\%$ and $16.1 \%$, respectively [142]. Other less common complications include pain (range $0 \%-7 \%$ ), bleeding (range $0 \%-6 \%$ ) $[13,18,42,101,139,151,171,177]$, tenesmus (up to $22 \%$, related to rectal SEMS), fever, incontinence, and fistula [22, $76,166,177-179]$.

Stent-related perforation may result from guidewire or catheter malpositioning, stricture dilation, stent-induced perforation, and proximal colonic distension because of inadequate colonic decompression or excessive air insufflation [180]. Reported rates of clinically evident early and late perforation range from $0 \%$ to $12 \%[17,76,77,83,86,101,105,110,124$, $139,143,147,151,168,169,171-173,175,176,181-189]$. A meta-analysis of 4086 patients reported an overall perforation rate of $7.4 \%$ [70]. In patients receiving stents as a bridge to surgery, "silent" microperforations may be identified in up to $14 \%$ $20 \%$, and pathologists should actively search for these in resection specimens $[102,103,105,115,168,189-191]$. Clinically symptomatic stent-related perforation has been associated with a mortality rate of $50 \%[17,77,81,151,168,192-195]$, and there are indications that perforation compromises oncological outcomes, although most studies were restricted by low numbers of events $[103,109,112,143,181]$. Intraprocedural and post-stenting stricture dilation, and longer, angulated, and diverticular strictures have been identified as risk factors for perforation $[18,21,44,63,67,70,77,81,123,154,170,196]$. Therefore, steps should be taken to avoid these situations where possible. Stent-related perforations are usually treated surgically $[101,169,190,197]$.

Median stent patency in the palliative setting ranges from 3 to 12 months (106 days in a systematic review) $[87,88,183$, 198-200], with approximately $50 \%$ patent at 12 months [194]. Stent patency is maintained in around $80 \%$ (range $53 \%$ $90 \%$ ) of patients until death or end of follow-up [64, 81, 86, 177 , 179, 201].

Migration rates range from $1 \%$ to $10 \%$, with some evidence that chemotherapy may be associated with higher rates because of tumor shrinkage $[58,61,76,83,86,101,103,104$, $139,151,165,169,171-173,175,184,189,192,202-205]$.
Stent occlusion due to overgrowth of malignant tissue, fecal impaction, or tumor ingrowth through the mesh occurs in 3\%$29 \%$ of cases, with higher rates reported in studies with longer follow-up and in cases of incomplete stent expansion $[61,83$, $86,143,171,173,175,184,196,206]$.

Both migration and re-obstruction can be managed by stent replacement or stent-in-stent techniques. These are reported as first choice in the majority of patients in the palliative setting, with satisfactory results (clinical success $75 \%-86 \%$ ) [74, 75], even though the long-term outcome is rarely reported. In the bridge-to-surgery setting, most patients with occluded or migrated stents are treated with earlier surgery, though the option of re-stenting remains and has not been compared to surgery in this setting $[17,21,61,64,83,175,177,178,196,198$, $206-208]$.

\section{Disclaimer}

ESGE Guidelines represent a consensus of best practice based on the available evidence at the time of preparation. They may not apply to all situations and should be interpreted in the setting of specific clinical situations and resource availability. They are intended to be an educational tool to provide information that may support endoscopists in providing care to patients. They are not rules and should not be utilized to establish a legal standard of care.

\section{Acknowledgment}

The authors would like to thank Cesare Hassan, Nuovo Regina Margherita Hospital, Rome, Italy, and Toshio Kuwai, National Hospital Organization, Kure Medical Center and Chugoku Cancer Center, Kure, Japan, for their thorough review of this Guideline.

\section{Competing interests}

S. Meisner provides consultancy for Olympus Europa (from April 2019, ongoing). G. Vanbliervliet has provided consultancy to Boston Scientific (2016 to present) and Cook Medical (2019 to present). J.E. van Hooft has received lecture fees from Medtronics (from 2014 to 2015 and 2019) and Cook Medical (2019) and consultancy fees from Boston Scientific (2014-2017); her department has received research grants from Cook Medical (2014-2019) and Abbott (20142017). D. Arnold, A. Arezzo R. Beets-Tan, S. Everett, M. Götz, E.E. van Halsema, J. Hill, G. Manes, E. Rodrigues-Pinto, C. Sabbagh, P.J. Tanis, J. Vandervoort and J.V. Veld have no competing interests.

\section{References}

[1] Jemal A, Bray F, Center MM et al. Global cancer statistics. CA Cancer ] Clin 2011; 61: 69-90

[2] Winner M, Mooney SJ, Hershman DL et al. Incidence and predictors of bowel obstruction in elderly patients with stage IV colon cancer: a population-based cohort study. JAMA Surg 2013; 148: 715-722

[3] Jullumstro E, Wibe A, Lydersen $\mathrm{S}$ et al. Colon cancer incidence, presentation, treatment and outcomes over 25 years. Colorectal Dis 2011; 13: 512-518 
[4] Cheynel N, Cortet M, Lepage C et al. Trends in frequency and management of obstructing colorectal cancers in a well-defined population. Dis Colon Rectum 2007; 50: 1568-1575

[5] Frago R, Ramirez E, Millan M et al. Current management of acute malignant large bowel obstruction: a systematic review. Am J Surg 2014; 207: $127-138$

[6] van Hooft JE, van Halsema EE, Vanbiervliet G et al. Self-expandable metal stents for obstructing colonic and extracolonic cancer: European Society of Gastrointestinal Endoscopy (ESGE) Clinical Guideline. Gastrointest Endosc 2014; 80: 747-761 , e1-75

[7] Fiori E, Lamazza A, De Cesare AA et al. Palliative management of malignant rectosigmoidal obstruction. Colostomy vs. endoscopic stenting. A randomized prospective trial. Anticancer Res 2004; 24: 265268

[8] Dumonceau JM, Hassan C, Riphaus A et al. European Society of Gastrointestinal Endoscopy (ESGE) Guideline Development Policy. Endoscopy 2012; 44: 626-629

[9] Wilkinson KJ, Chua W, Ng W et al. Management of asymptomatic primary tumours in stage IV colorectal cancer: Review of outcomes. World J Gastrointest Oncol 2015; 7: 513-523

[10] Matsumoto T, Hasegawa S, Matsumoto $S$ et al. Overcoming the challenges of primary tumor management in patients with metastatic colorectal cancer unresectable for cure and an asymptomatic primary tumor. Dis Colon Rectum 2014; 57: 679-686

[11] Suarez J, Marin G, Vera R et al. Complications from the primary tumour are not related with survival in patients with synchronous stage IV colorectal cancer receiving chemotherapy without primary tumour resection. Int J Colorectal Dis 2015; 30: 1357-1363

[12] Kim DH, Kim B, Choi JH et al. Tumor characteristics associated with malignant large bowel obstruction in stage IV colorectal cancer patients undergoing chemotherapy. Int J Colorectal Dis 2016; 31: 17671774

[13] Yoon JY, Jung YS, Hong SP et al. Clinical outcomes and risk factors for technical and clinical failures of self-expandable metal stent insertion for malignant colorectal obstruction. Gastrointest Endosc 2011; 74: 858-868

[14] Kim JH, Ku YS, Jeon TJ et al. The efficacy of self-expanding metal stents for malignant colorectal obstruction by noncolonic malignancy with peritoneal carcinomatosis. Dis Colon Rectum 2013; 56: 1228-1232

[15] Song HY, Kim JH, Kim KR et al. Malignant rectal obstruction within 5 $\mathrm{cm}$ of the anal verge: is there a role for expandable metallic stent placement? Gastrointest Endosc 2008; 68: 713-720

[16] Japan Colonic Stent Safe Procedure Research (JCSSPR) Group. CROSS: ColoRectal Obstruction Scoring System 2012. Available from: https:// colon-stent.com/001_mainpage_en.html

[17] Abbott S, Eglinton TW, Ma Y et al. Predictors of outcome in palliative colonic stent placement for malignant obstruction. Br J Surg 2014; 101: 121-126

[18] Meisner S, Gonzalez-Huix F, Vandervoort JG et al. Self-expandable metal stents for relieving malignant colorectal obstruction: shortterm safety and efficacy within 30 days of stent procedure in 447 patients. Gastrointest Endosc 2011; 74: 876-884

[19] Choi JH, Lee YJ, Kim ES et al. Covered self-expandable metal stents are more associated with complications in the management of malignant colorectal obstruction. Surg Endosc 2013; 27: 3220-3227

[20] Donnellan F, Cullen G, Cagney D et al. Efficacy and safety of colonic stenting for malignant disease in the elderly. Int J Colorectal Dis 2010; 25: 747-750

[21] Small A], Coelho-Prabhu N, Baron TH. Endoscopic placement of selfexpandable metal stents for malignant colonic obstruction: longterm outcomes and complication factors. Gastrointest Endosc 2010; 71: $560-572$
[22] Geraghty J, Sarkar S, Cox T et al. Management of large bowel obstruction with self-expanding metal stents. A multicentre retrospective study of factors determining outcome. Colorectal Dis 2014; 16: 476-483

[23] Biondo S, Pares D, Frago R et al. Large bowel obstruction: predictive factors for postoperative mortality. Dis Colon Rectum 2004; 47: 1889-1897

[24] Tekkis PP, Kinsman R, Thompson MR et al. The Association of Coloproctology of Great Britain and Ireland study of large bowel obstruction caused by colorectal cancer. Ann Surg 2004; 240: 76-81

[25] Tan KK, Sim R. Surgery for obstructed colorectal malignancy in an Asian population: predictors of morbidity and comparison between left- and right-sided cancers. J Gastrointest Surg 2010; 14: 295-302

[26] Frager D, Rovno HD, Baer JW et al. Prospective evaluation of colonic obstruction with computed tomography. Abdom Imaging 1998; 23: 141-146

[27] Ngu J, Lieske B, Chan KH et al. Caecal pneumatosis is not an absolute contraindication for endoluminal stenting in patients with acute malignant large bowel obstruction. ANZ J Surg 2014; 84: 772-775

[28] Kodeda K, Nathanaelsson L, Jung B et al. Population-based data from the Swedish Colon Cancer Registry. Br J Surg 2013; 100: 1100-1107

[29] Latournerie M, Jooste V, Cottet V et al. Epidemiology and prognosis of synchronous colorectal cancers. Br J Surg 2008; 95: 1528-1533

[30] Mulder SA, Kranse R, Damhuis RA et al. Prevalence and prognosis of synchronous colorectal cancer: a Dutch population-based study. Cancer Epidemiol 2011; 35: 442-447

[31] Papadopoulos V, Michalopoulos A, Basdanis G et al. Synchronous and metachronous colorectal carcinoma. Techn Coloproctol 2004; 8: (Suppl. 01): s97-s100

[32] Maruo H, Tsuyuki $\mathrm{H}$, Kojima T et al. [Findings from total colonoscopy in obstructive colorectal cancer patients who underwent stent placement as a bridge to surgery (BTS)]. Gan To Kagaku Ryoho 2017; 44: $1238-1240$

[33] Min CK, Kim HO, Lee D et al. Obstructive left colon cancer should be managed by using a subtotal colectomy instead of colonic stenting. Ann Coloproctol 2016; 32: 215-220

[34] Kim JS, Lee KM, Kim SW et al. Preoperative colonoscopy through the colonic stent in patients with colorectal cancer obstruction. World ] Gastroenterol 2014; 20: 10570-10576

[35] Yanar H, Ozcinar B, Yanar F et al. The role of colorectal stent placement in the management of acute malignant obstruction. Ulus Travma Acil Cerrahi Derg [Turkish J Trauma Emerg Surg TJTES] 2014; 20: 23-27

[36] Singh K, Narula AK, Thukral CL et al. H. Role of CT colonography in colonic lesions and its correlation with conventional colonoscopic findings. J Clin Diagn Res 2015; 9: TC14-18

[37] Huisman JF, Leicher LW, de Boer E et al. Consequences of CT colonography in stenosing colorectal cancer. Int J Colorectal Dis 2017; 32: 367-373

[38] Kim WS, Lee HS, Lee JM et al. Fluoro-2-deoxy-d-glucose positron emission tomography/computed tomography for the detection of proximal synchronous lesions in patients with obstructive colorectal cancer. J Gastroenterol Hepatol 2017; 32: 401-408

[39] Maeda C, Endo S, Mori Y et al. The ability of positron emission tomography/computed tomography to detect synchronous colonic cancers in patients with obstructive colorectal cancer. Mol Clin Oncol 2019; 10: 425-429

[40] Hojo D, Tanaka T, Takahashi M et al. Efficacy of 18-fluoro deoxy glucose-positron emission tomography computed tomography for the detection of colonic neoplasia proximal to obstructing colorectal cancer. Medicine 2018; 97: e11655 
[41] Pirlet IA, Slim K, Kwiatkowski F et al. Emergency preoperative stenting versus surgery for acute left-sided malignant colonic obstruction: a multicenter randomized controlled trial. Surg Endosc 2011; 25: 1814-1821

[42] van Hooft JE, Bemelman WA, Oldenburg B et al. Colonic stenting versus emergency surgery for acute left-sided malignant colonic obstruction: a multicentre randomised trial. Lancet Oncol 2011; 12: 344-352

[43] Sai VF, Velayos F, Neuhaus J et al. Colonoscopy after CT diagnosis of diverticulitis to exclude colon cancer: a systematic literature review. Radiology 2012; 263: 383-390

[44] Currie A, Christmas C, Aldean H et al. Systematic review of self-expanding stents in the management of benign colorectal obstruction. Colorectal Dis 2014; 16: 239-245

[45] Brouwer R, MacDonald A, Matthews R et al. Brush cytology for the diagnosis of colorectal cancer. Dis Colon Rectum 2009; 52: 598-601

[46] Geramizadeh B, Hooshmand F, Kumar PV. Brush cytology of colorectal malignancies. Acta Cytol 2003; 47: 431-434

[47] Farouk R, Edwards J, Thorne M et al. Brush cytology for the diagnosis of rectal carcinoma. Br J Surg 1996; 83: 1456-1458

[48] Kuwai T, Yamaguchi T, Imagawa $\mathrm{H}$ et al. Factors related to difficult self-expandable metallic stent placement for malignant colonic obstruction: A post-hoc analysis of a multicenter study across Japan. Dig Endosc 2019; 31: 51-58

[49] Chun Y], Yoon NR, Park JM et al. Prospective assessment of risk of bacteremia following colorectal stent placement. Dig Dis Sci 2012; 57: 1045-1049

[50] Kim JS, Lee WS, Chung CY et al. Clinical impact of prophylactic antibiotic treatment for self-expandable metallic stent insertion in patients with malignant colorectal obstruction. Gastroenterol Res Pract 2015; 2015: 416142

[51] Williams D, Law R, Pullyblank AM. Colorectal stenting in malignant large bowel obstruction: the learning curve. Int J Surg Oncol 2011; 2011: 917848

[52] Lee JH, Yoon JY, Park S] et al. The learning curve for colorectal stent insertion for the treatment of malignant colorectal obstruction. Gut Liver 2012; 6: 328-333

[53] Lee H], Park S], Cheon JH et al. What is the necessity of endoscopist for successful endoscopic stenting in patients with malignant colorectal obstruction? Int J Colorectal Dis 2015; 30: 119-125

[54] Tang MH, Ross T, Yeo SA et al. Colonic stenting for malignant large bowel obstruction is safe and effective: a single-surgeon experience. Singapore Med J 2019; 60: 347-352

[55] Kim SY, Kwon SH, Oh JH. Radiologic placement of uncovered stents for the treatment of malignant colorectal obstruction. J Vasc Interv Radiol 2010; 21: 1244-1249

[56] Kim H, Kim SH, Choi SY et al. Fluoroscopically guided placement of self-expandable metallic stents and stent-grafts in the treatment of acute malignant colorectal obstruction. J Vasc Interv Radiol 2008; 19 : 1709-1716

[57] Shrivastava V, Tariq O, Tiam R et al. Palliation of obstructing malignant colonic lesions using self-expanding metal stents: a single-center experience. Cardiovasc Interv Radiol 2008; 31: 931-936

[58] Kim JH, Song HY, Li YD et al. Dual-design expandable colorectal stent for malignant colorectal obstruction: comparison of flared ends and bent ends. AJR Am J Roentgenol 2009; 193: 248-254

[59] Alcantara M, Serra X, Bombardo J et al. Colorectal stenting as an effective therapy for preoperative and palliative treatment of large bowel obstruction: 9 years' experience. Techn Coloproctol 2007; 11: $316-322$

[60] Selinger CP, Ramesh J, Martin DF. Long-term success of colonic stent insertion is influenced by indication but not by length of stent or site of obstruction. Int J Colorectal Dis 2011; 26: 215-218
[61] Yoon J, Kwon SH, Lee CK et al. Radiologic placement of uncovered stents for the treatment of malignant colonic obstruction proximal to the descending colon. Cardiovasc Interv Radiol 2017; 40: 99-105

[62] Kim JW, Jeong JB, Lee KL et al. Comparison of clinical outcomes between endoscopic and radiologic placement of self-expandable metal stent in patients with malignant colorectal obstruction. Korean J Gastroenterol 2013; 61: 22-29

[63] Sebastian S, Johnston S, Geoghegan T et al. Pooled analysis of the efficacy and safety of self-expanding metal stenting in malignant colorectal obstruction. Am J Gastroenterol 2004; 99: 2051-2057

[64] de Gregorio MA, Laborda A, Tejero E et al. Ten-year retrospective study of treatment of malignant colonic obstructions with self-expandable stents. J Vasc Interv Radiol 2011; 22: 870-878

[65] Rodrigues-Pinto E, Pereira P, Lopes S et al. Outcome of endoscopic self-expandable metal stents in acute malignant colorectal obstruction at a tertiary center. Rev Esp Enferm Dig 2015; 107: 534-538

[66] Samper Wamba JD, Fernandez Martinez A, Gonzalez Pastrana L et al. Efficacy and complications in the use of self-expanding colonic stents: an analysis of 15 years' experience. Radiologia 2015; 57: 402-411

[67] Abbas MA, Kharabadze G, Ross EM et al. Predictors of outcome for endoscopic colorectal stenting: a decade experience. Int J Colorectal Dis 2017; 32: 375-382

[68] Tanaka A, Sadahiro S, Yasuda M et al. Endoscopic balloon dilation for obstructive colorectal cancer: a basic study on morphologic and pathologic features associated with perforation. Gastrointest Endosc 2010; 71: 799-805

[69] Khot UP, Lang AW, Murali K et al. Systematic review of the efficacy and safety of colorectal stents. Br J Surg 2002; 89: 1096-1102

[70] van Halsema EE, van Hooft JE, Small A] et al. Perforation in colorectal stenting: a meta-analysis and a search for risk factors. Gastrointest Endosc 2014; 79: 970-82.e7 ; quiz 83.e2, 83.e5

[71] Mashar M, Mashar R, Hajibandeh S. Uncovered versus covered stent in management of large bowel obstruction due to colorectal malignancy: a systematic review and meta-analysis. Int J Colorectal Dis 2019; 34: 773-785

[72] Zhang Y, Shi J, Shi B et al. Comparison of efficacy between uncovered and covered self-expanding metallic stents in malignant large bowel obstruction: a systematic review and meta-analysis. Colorectal Dis 2012; 14: e367-e374

[73] Yang Z, Wu Q, Wang F et al. A systematic review and meta-analysis of randomized trials and prospective studies comparing covered and bare self-expandable metal stents for the treatment of malignant obstruction in the digestive tract. Int J Med Sci 2013; 10: 825-835

[74] Yoon JY, Park S], Hong SP et al. Outcomes of secondary self-expandable metal stents versus surgery after delayed initial palliative stent failure in malignant colorectal obstruction. Digestion 2013; 88: 4655

[75] Yoon JY, Jung YS, Hong SP et al. Outcomes of secondary stent-in-stent self-expandable metal stent insertion for malignant colorectal obstruction. Gastrointest Endosc 2011; 74: 625-633

[76] Tomita M, Saito S, Makimoto $S$ et al. Self-expandable metallic stenting as a bridge to surgery for malignant colorectal obstruction: pooled analysis of 426 patients from two prospective multicenter series. Surg Endosc 2019; 33: 499-509

[77] Lee Y], Yoon JY, Park JJ et al. Clinical outcomes and factors related to colonic perforations in patients receiving self-expandable metal stent insertion for malignant colorectal obstruction. Gastrointest Endosc 2018; 87: 1548-1557.e1

[78] Matsuda A, Miyashita M, Matsumoto S et al. Optimal interval from placement of a self-expandable metallic stent to surgery in patients with malignant large bowel obstruction: A preliminary study. Surg Laparosc Endosc Percutan Tech 2018; 28: 239-244 
[79] Ogawa S, Ishii T, Minaga K et al. The feasibility of 18-mm-diameter colonic stents for obstructive colorectal cancers. Oncology 2017; 93 : (Suppl. 01): 43-48

[80] Kim BC, Han KS, Hong CW et al. Clinical outcomes of palliative selfexpanding metallic stents in patients with malignant colorectal obstruction. J Dig Dis 2012; 13: 258-266

[81] Manes G, de Bellis M, Fuccio L et al. Endoscopic palliation in patients with incurable malignant colorectal obstruction by means of self-expanding metal stent: analysis of results and predictors of outcomes in a large multicenter series. Arch Surg 2011; 146: 1157-1162

[82] Im JP, Kim SG, Kang HW et al. Clinical outcomes and patency of selfexpanding metal stents in patients with malignant colorectal obstruction: a prospective single center study. Int J Colorectal Dis 2008; 23: 789-794

[83] Imai M, Kamimura K, Takahashi Y et al. The factors influencing longterm outcomes of stenting for malignant colorectal obstruction in elderly group in community medicine. Int J Colorectal Dis 2018; 33 : 189-197

[84] Pais-Cunha I, Castro R, Libanio D et al. Endoscopic stenting for palliation of intra-abdominal gastrointestinal malignant obstruction: predictive factors for clinical success. Eur ] Gastroenterol Hepatol 2018; 30: $1033-1040$

[85] van den Berg EH, Bergmann JF, Ledeboer M et al. Radiological position and clinical outcome of preoperative self-expanding metal stents for obstructing colonic cancer: a single-centre cohort study. Dig Surg 2015; 32: 262-268

[86] Di Mitri R, Mocciaro F, Traina M et al. Self-expandable metal stents for malignant colonic obstruction: data from a retrospective regional SIED-AIGO study. Dig Liver Dis 2014; 46: 279-282

[87] Cheung DY, Kim JY, Hong SP et al. Outcome and safety of self-expandable metallic stents for malignant colon obstruction: a Korean multicenter randomized prospective study. Surg Endosc 2012; 26: 3106-3113

[88] Park JK, Lee MS, Ko BM et al. Outcome of palliative self-expanding metal stent placement in malignant colorectal obstruction according to stent type and manufacturer. Surg Endosc 2011; 25: 1293-1299

[89] Small AJ, Baron TH. Comparison of Wallstent and Ultraflex stents for palliation of malignant left-sided colon obstruction: a retrospective, case-matched analysis. Gastrointest Endosc 2008; 67: 478-488

[90] Cheung HY, Chung CC, Tsang WW et al. Endolaparoscopic approach vs conventional open surgery in the treatment of obstructing left-sided colon cancer: a randomized controlled trial. Arch Surg 2009; 144 : $1127-1132$

[91] Alcantara M, Serra-Aracil X, Falco J et al. Prospective, controlled, randomized study of intraoperative colonic lavage versus stent placement in obstructive left-sided colonic cancer. World J Surg 2011; 35 : 1904-1910

[92] Ho KS, Quah HM, Lim JF et al. Endoscopic stenting and elective surgery versus emergency surgery for left-sided malignant colonic obstruction: a prospective randomized trial. Int J Colorectal Dis 2012; 27: $355-362$

[93] Ghazal AH, El-Shazly WG, Bessa SS et al. Colonic endolumenal stenting devices and elective surgery versus emergency subtotal/total colectomy in the management of malignant obstructed left colon carcinoma. J Gastrointest Surg 2013; 17: 1123-1129

[94] Arezzo A, Balague C, Targarona E et al. Colonic stenting as a bridge to surgery versus emergency surgery for malignant colonic obstruction: results of a multicentre randomised controlled trial (ESCO trial). Surg Endosc 2017; 31: 3297-3305

[95] Cui], Zhang JL, Wang $S$ et al. [A preliminary study of stenting followed by laparoscopic surgery for obstructing left-sided colon cancer] [Article in Chinese]. Zhonghua Wei Chang Wai Ke Za Zhi 2011; 14: 40-43
[96] Hill J, Kay C, Morton D et al. CREST: Randomised phase III study of stenting as a bridge to surgery in obstructing colorectal cancer - Results of the UK ColoRectal Endoscopic Stenting Trial (CREST). J Clin Oncol 2016; 34 : (Suppl. 15): 3507

[97] Amelung F], Consten EC], Siersema PD et al. A population-based analysis of three treatment modalities for malignant obstruction of the proximal colon: acute resection versus stent or stoma as a bridge to surgery. Ann Surg Oncol 2016; 23: 3660-3668

[98] Amelung F], Draaisma WA, Consten EC] et al. Self-expandable metal stent placement versus emergency resection for malignant proximal colon obstructions. Surg Endosc 2017; 31: 4532-4541

[99] Amelung F], de Beaufort HW, Siersema PD et al. Emergency resection versus bridge to surgery with stenting in patients with acute right-sided colonic obstruction: a systematic review focusing on mortality and morbidity rates. Int J Colorectal Dis 2015; 30: 1147-1155

[100] Amelung FJ, Burghgraef TA, Tanis PJ et al. Critical appraisal of oncological safety of stent as bridge to surgery in left-sided obstructing colon cancer; a systematic review and meta-analysis. Crit Rev Oncol Hematol 2018; 131: 66-75

[101] Rodrigues-Pinto E, Morais R, Coelho C et al. Bridge-to-surgery versus emergency surgery in the management of left-sided acute malignant colorectal obstruction - Efficacy, safety and long-term outcomes. Dig Liver Dis 2019; 51: 364-372

[102] Mege D, Sabbagh C, Manceau G et al. What is the best option between primary diverting stoma or endoscopic stent as a bridge to surgery with a curative intent for obstructed left colon cancer? Results from a propensity score analysis of the French Surgical Association multicenter cohort of 518 patients Ann Surg Oncol 2019; 26: 756-764

[103] Verstockt B, Van Driessche A, De Man M et al. Ten-year survival after endoscopic stent placement as a bridge to surgery in obstructing colon cancer. Gastrointest Endosc 2018; 87: 705-713.e2

[104] Flor-Lorente B, Baguena G, Frasson M et al. Self-expanding metallic stent as a bridge to surgery in the treatment of left colon cancer obstruction: Cost-benefit analysis and oncologic results. Cir Esp 2017; 95: 143-151

[105] Saito S, Yoshida S, Isayama $\mathrm{H}$ et al. A prospective multicenter study on self-expandable metallic stents as a bridge to surgery for malignant colorectal obstruction in Japan: efficacy and safety in $312 \mathrm{pa}-$ tients. Surg Endosc 2016; 30: 3976-3986

[106] Li CY, Guo SB, Wang NF. Decompression of acute left-sided malignant colorectal obstruction: comparing transanal drainage tube with metallic stent. J Clin Gastroenterol 2014; 48: e37-42

[107] Foo CC, Poon SHT, Chiu RHY et al. Is bridge to surgery stenting a safe alternative to emergency surgery in malignant colonic obstruction: a meta-analysis of randomized control trials. Surg Endosc 2019; 33: 293-302

[108] Yang P, Lin XF, Lin K et al. The role of stents as bridge to surgery for acute left-sided obstructive colorectal cancer: meta-analysis of randomized controlled trials. Rev Invest Clin 2018; 70: 269-278

[109] Sloothaak DA, van den Berg MW, Dijkgraaf MG et al. Oncological outcome of malignant colonic obstruction in the Dutch Stent-In 2 trial. Br J Surg 2014; 101: 1751-1757

[110] Amelung F], Borstlap WAA, Consten EC] et al. Propensity score-matched analysis of oncological outcome between stent as bridge to surgery and emergency resection in patients with malignant left-sided colonic obstruction. Br J Surg 2019; 106: 1075-1086

[111] Maruthachalam K, Lash GE, Shenton BK et al. Tumour cell dissemination following endoscopic stent insertion. Br J Surg 2007; 94 : 1151-1154

[112] Sabbagh C, Browet F, Diouf M et al. Is stenting as "a bridge to surgery" an oncologically safe strategy for the management of acute, left-sided, malignant, colonic obstruction? A comparative study with a propensity score analysis. Ann Surg 2013; 258: 107-115 
[113] Kim H], Choi GS, Park JS et al. Higher rate of perineural invasion in stent-laparoscopic approach in comparison to emergent open resection for obstructing left-sided colon cancer. Int J Colorectal Dis 2013; 28: 407-414

[114] Matsuda A, Yamada T, Matsumoto S et al. Short-term outcomes of a self-expandable metallic stent as a bridge to surgery vs. a transanal decompression tube for malignant large-bowel obstruction: a metaanalysis. Surg Today 2019; 49: 728-737

[115] Allievi N, Ceresoli M, Fugazzola P et al. Endoscopic stenting as bridge to surgery versus emergency resection for left-sided malignant colorectal obstruction: an updated meta-analysis. Int J Surg Oncol 2017; 2017: 2863272

[116] Arezzo A, Passera R, Lo Secco G et al. Stent as bridge to surgery for left-sided malignant colonic obstruction reduces adverse events and stoma rate compared with emergency surgery: results of a systematic review and meta-analysis of randomized controlled trials. Gastrointest Endosc 2017; 86: 416-426

[117] Wang X, He J, Chen X et al. Stenting as a bridge to resection versus emergency surgery for left-sided colorectal cancer with malignant obstruction: A systematic review and meta-analysis. Int J Surg 2017; 48: 64-68

[118] Huang X, Lv B, Zhang S et al. Preoperative colonic stents versus emergency surgery for acute left-sided malignant colonic obstruction: a meta-analysis. J Gastrointest Surg 2014; 18: 584-591

[119] Zhao X, Liu B, Zhao E et al. The safety and efficiency of surgery with colonic stents in left-sided malignant colonic obstruction: a metaanalysis. Gastroenterol Res Pract 2014; 2014: 407325

[120] Ceresoli M, Allievi N, Coccolini F et al. Long-term oncologic outcomes of stent as a bridge to surgery versus emergency surgery in malignant left side colonic obstructions: a meta-analysis. J Gastrointest Oncol 2017; 8: 867-876

[121] Matsuda A, Miyashita M, Matsumoto S et al. Comparison of longterm outcomes of colonic stent as "bridge to surgery" and emergency surgery for malignant large-bowel obstruction: a meta-analysis. Ann Surg Oncol 2015; 22: 497-504

[122] Schoonbeek PK, Genzel P, van den Berg EH et al. Outcomes of selfexpanding metal stents in malignant colonic obstruction are independent of location or length of the stenosis: results of a retrospective, single-center series. Dig Surg 2018; 35: 230-235

[123] Boyle DJ, Thorn C, Saini A et al. Predictive factors for successful colonic stenting in acute large-bowel obstruction: a 15-year cohort analysis. Dis Colon Rectum 2015; 58: 358-362

[124] Matsuzawa T, Ishida H, Yoshida S et al. A Japanese prospective multicenter study of self-expandable metal stent placement for malignant colorectal obstruction: short-term safety and efficacy within 7 days of stent procedure in 513 cases. Gastrointest Endosc 2015; 82: 697-707.e1

[125] Abdussamet Bozkurt M, Gonenc M, Kapan S et al. Colonic stent as bridge to surgery in patients with obstructive left-sided colon cancer. JSLS 2014; 18: pii: e2014.00161 doi:10.4293/JSLS.2014.00161

[126] Sirikurnpiboon S, Awapittaya B, Jivapaisarnpong P et al. Bridging metallic stent placement in acute obstructed left sided malignant colorectal cancer: optimal time for surgery. J Med Assoc Thai 2014; 97: (Suppl. 11): S81-S86

[127] Kim JH, Kwon KA, Lee JJ et al. Surgical failure after colonic stenting as a bridge to surgery. World J Gastroenterol 2014; 20: 11826-11834

[128] Ho KM, Chan KM, Kwok SY et al. Colonic self-expanding metal stent (SEMS) as a bridge to surgery in left-sided malignant colonic obstruction: an 8-year review. Surg Endosc 2017; 31: 2255-2262

[129] Broholm M, Kobborg M, Frostberg E et al. Delay of surgery after stent placement for resectable malignant colorectal obstruction is associated with higher risk of recurrence. Int J Colorectal Dis 2017; 32: $513-516$
[130] Lee G], Kim H], Baek JH et al. Comparison of short-term outcomes after elective surgery following endoscopic stent insertion and emergency surgery for obstructive colorectal cancer. Int I Surg 2013; 11: 442-446

[131] Seymour MT, Morton D. FOxTROT: an international randomised controlled trial in 1052 patients evaluating neoadjuvant chemotherapy (NAC) for colon cancer. J Clin Oncol 2019; 37: Abstract 3504 doi:10.1200/JCO.2019.37.15_suppl.3504

[132] Veld JV, Amelung FJ, Borstlap WAA et al. Comparison of decompressing stoma vs stent as a bridge to surgery for left-sided obstructive colon cancer. JAMA Surg 2020: doi:10.1001/jamasurg.2019.5466 Epub ahead of print

[133] Oistamo E, Hjern F, Blomqvist L et al. Emergency management with resection versus proximal stoma or stent treatment and planned resection in malignant left-sided colon obstruction. World J Surg Oncol 2016; 14: 232

[134] Amelung F], Ter Borg F, Consten EC et al. Deviating colostomy construction versus stent placement as bridge to surgery for malignant left-sided colonic obstruction. Surg Endosc 2016; 30: 5345-5355

[135] Cho YK, Kim SW, Lee BI et al. Clinical outcome of self-expandable metal stent placement in the management of malignant proximal colon obstruction. Gut Liver 2011; 5: 165-170

[136] Yao LQ, Zhong YS, Xu MD et al. Self-expanding metallic stents drainage for acute proximal colon obstruction. World J Gastroenterol 2011; 17: 3342-3346

[137] Repici A, Adler DG, Gibbs CM et al. Stenting of the proximal colon in patients with malignant large bowel obstruction: techniques and outcomes. Gastrointest Endosc 2007; 66: 940-944

[138] Dronamraju SS, Ramamurthy S, Kelly SB et al. Role of self-expanding metallic stents in the management of malignant obstruction of the proximal colon. Dis Colon Rectum 2009; 52: 1657-1661

[139] Morita S, Yamamoto K, Ogawa A et al. Benefits of using a selfexpandable metallic stent as a bridge to surgery for right- and leftsided obstructive colorectal cancers. Surg Today 2019; 49: 32-37

[140] Kye BH, Lee YS, Cho HM et al. Comparison of long-term outcomes between emergency surgery and bridge to surgery for malignant obstruction in right-sided colon cancer: a multicenter retrospective study. Ann Surg Oncol 2016; 23: 1867-1874

[141] Siddiqui A, Cosgrove N, Yan LH et al. Long-term outcomes of palliative colonic stenting versus emergency surgery for acute proximal malignant colonic obstruction: a multicenter trial. Endosc Int Open 2017; 5: e232-e238

[142] Liang TW, Sun Y, Wei YC et al. Palliative treatment of malignant colorectal obstruction caused by advanced malignancy: a self-expanding metallic stent or surgery? A system review and meta-analysis Surg Today 2014; 44: 22-33

[143] Ribeiro IB, Bernardo WM, Martins BDC et al. Colonic stent versus emergency surgery as treatment of malignant colonic obstruction in the palliative setting: a systematic review and meta-analysis. Endosc Int Open 2018; 6: e558-e567

[144] Takahashi H, Okabayashi K, Tsuruta M et al. Self-expanding metallic stents versus surgical intervention as palliative therapy for obstructive colorectal cancer: a meta-analysis. World J Surg 2015; 39: 2037 2044

[145] Zhao XD, Cai BB, Cao RS et al. Palliative treatment for incurable malignant colorectal obstructions: a meta-analysis. World J Gastroenterol 2013; 19: 5565-5574

[146] Abelson JS, Yeo HL, Mao J et al. A long-term postprocedural outcomes of palliative emergency stenting vs stoma in malignant largebowel obstruction. JAMA Surg 2017; 152: 429-435

[147] Young C], De-Loyde KJ, Young JM et al. Improving quality of life for people with incurable large-bowel obstruction: randomized control trial of colonic stent insertion. Dis Colon Rectum 2015; 58: 838-849 
[148] Karoui M, Charachon A, Delbaldo C et al. Stents for palliation of obstructive metastatic colon cancer: impact on management and chemotherapy administration. Arch Surg 2007; 142: 619-623; discussion 623

[149] Young C], Zahid A. Randomized controlled trial of colonic stent insertion in non-curable large bowel obstruction: a post hoc cost analysis. Colorectal Dis 2018; 20: 288-295

[150] Faraz S, Salem SB, Schattner M et al. Predictors of clinical outcome of colonic stents in patients with malignant large-bowel obstruction because of extracolonic malignancy. Gastrointest Endosc 2018; 87 : 1310-1317

[151] Park YE, Park Y, Park S] et al. Outcomes of stent insertion and mortality in obstructive stage IV colorectal cancer patients through 10 year duration. Surg Endosc 2019; 33: 1225-1234

[152] Park JJ, Rhee K, Yoon JY et al. Impact of peritoneal carcinomatosis on clinical outcomes of patients receiving self-expandable metal stents for malignant colorectal obstruction. Endoscopy 2018; 50: 11631174

[153] Ceze N, Charachon A, Locher C et al. Safety and efficacy of palliative systemic chemotherapy combined with colorectal self-expandable metallic stents in advanced colorectal cancer: A multicenter study. Clin Res Hepatol Gastroenterol 2016; 40: 230-238

[154] Imbulgoda A, MacLean A, Heine J et al. Colonic perforation with intraluminal stents and bevacizumab in advanced colorectal cancer: retrospective case series and literature review. Can J Surg 2015; 58 : 167-171

[155] Bong JW, Lee JL, Kim CW et al. Risk factors and adequate management for complications of bevacizumab treatment requiring surgical intervention in patients with metastatic colorectal cancer. Clin Colorectal Cancer 2018; 17: e639-e645

[156] Kim JY, Kim SG, Im JP et al. Comparison of treatment outcomes of endoscopic stenting for colonic and extracolonic malignant obstruction. Surg Endosc 2013; 27: 272-277

[157] Moon S], Kim SW, Lee BI et al. Palliative stent for malignant colonic obstruction by extracolonic malignancy: a comparison with colorectal cancer. Dig Dis Sci 2014; 59: 1891-1897

[158] Kim BK, Hong SP, Heo HM et al. Endoscopic stenting is not as effective for palliation of colorectal obstruction in patients with advanced gastric cancer as emergency surgery. Gastrointest Endosc 2012; 75 : 294-301

[159] Kim JH, Song HY, Park JH et al. Metallic stent placement in the palliative treatment of malignant colonic obstructions: primary colonic versus extracolonic malignancies. J Vasc Intervent Radiol 2011; 22: $1727-1732$

[160] Trompetas V, Saunders M, Gossage J et al. Shortcomings in colonic stenting to palliate large bowel obstruction from extracolonic malignancies. Int J Colorectal Dis 2010; 25: 851-854

[161] Shin SJ, Kim TI, Kim BC et al. Clinical application of self-expandable metallic stent for treatment of colorectal obstruction caused by extrinsic invasive tumors. Dis Colon Rectum 2008; 51: 578-583

[162] Keranen I, Lepisto A, Udd M et al. Stenting for malignant colorectal obstruction: a single-center experience with 101 patients. Surg Endosc 2012; 26: 423-430

[163] Keswani RN, Azar RR, Edmundowicz SA et al. Stenting for malignant colonic obstruction: a comparison of efficacy and complications in colonic versus extracolonic malignancy. Gastrointest Endosc 2009; $69: 675-680$

[164] Jutzi L, Russell D, Ho S et al. The role of palliative colorectal stents in gynaecologic malignancy. Gynecol Oncol 2014; 134: 566-569

[165] Bayraktar B, Ozemir IA, Kefeli U et al. Colorectal stenting for palliation and as a bridge to surgery: A 5-year follow-up study. World J Gastroenterol 2015; 21: 9373-9379
[166] Gallo G, Sammarco G, Chiriatti AP et al. The role of self-expandable metallic stents as "bridge to surgery" for the treatment of acute malignant colorectal obstruction. Our experience. Ann Ital Chir 2017; 6: 418-424

[167] Gargallo C], Ferrandez A, Carrera P et al. Short- and long-term clinical outcomes of self-expandable metal stents inserted for colorectal obstruction and efficacy of different insertion techniques. Gastroenterol Hepatol 2019; 42: 157-163

[168] Gleditsch D, Soreide OK, Nesbakken A. Managing malignant colorectal obstruction with self-expanding stents. a closer look at bowel perforations and failed procedures. J Gastrointest Surg 2016; 20: 1643-1649

[169] Kobborg M, Broholm M, Frostberg E et al. Short-term results of selfexpanding metal stents for acute malignant large bowel obstruction. Colorectal Dis 2017; 19: 0365-0371

[170] Kohler G, Antoniou SA, Lechner M et al. Stenting for emergency colorectal obstruction: an analysis of 204 patients in relation to predictors of failure and complications. Scand J Surg 2015; 104: 146-153

[171] Lee H], Hong SP, Cheon JH et al. Clinical Outcomes of self-expandable metal stents for malignant rectal obstruction. Dis Colon Rectum 2018; 61: 43-50

[172] Lim TZ, Chan DKH, Tan KK. Endoscopic stenting does not worsen long term outcomes amongst patients presenting with obstruction from colorectal cancers. Ann Surg Oncol 2017; 24: 1618-1625

[173] Park S], Lee KY, Kwon SH et al. Stenting as a bridge to surgery for obstructive colon cancer: does it have surgical merit or oncologic demerit? Ann Surg Oncol 2016; 23: 842-848

[174] Salamone G, Falco N, Atzeni ] et al. Colonic stenting in acutely obstructed left-sided colon cancer Clinical evaluation and cost analysis. Ann Ital Chir 2014; 85: 556-562

[175] Sousa M, Pinho R, Proenca L et al. Predictors of complications and mortality in patients with self-expanding metallic stents for the palliation of malignant colonic obstruction. GE Port J Gastroenterol 2017; 24: 122-128

[176] Mehmood RK, Parker J, Kirkbride P et al. Outcomes after stenting for malignant large bowel obstruction without radiologist support. World J Gastroenterol 2014; 20: 6309-6313

[177] Gianotti L, Tamini N, Nespoli L et al. A prospective evaluation of short-term and long-term results from colonic stenting for palliation or as a bridge to elective operation versus immediate surgery for large-bowel obstruction. Surg Endosc 2013; 27: 832-842

[178] Angenete E, Asplund D, Bergstrom M et al. Stenting for colorectal cancer obstruction compared to surgery-a study of consecutive patients in a single institution. Int J Colorectal Dis 2012; 27: 665670

[179] Young C], Suen MK, Young J et al. Stenting large bowel obstruction avoids a stoma: consecutive series of 100 patients. Colorectal Dis 2011; 13: 1138-1141

[180] Baron TH, Wong Kee Song LM, Repici A. Role of self-expandable stents for patients with colon cancer (with videos). Gastrointest Endosc 2012; 75: 653-662

[181] Avlund TH, Erichsen R, Ravn S et al. The prognostic impact of bowel perforation following self-expanding metal stent as a bridge to surgery in colorectal cancer obstruction. Surg Endosc 2018; 32: 328336

[182] Kim MK, Kye BH, Lee IK et al. Outcome of bridge to surgery stenting for obstructive left colon cancer. ANZ J Surg 2017; 87: e245-e250

[183] Lee HJ, Park S], Min BS et al. The role of primary colectomy after successful endoscopic stenting in patients with obstructive metastatic colorectal cancer. Dis Colon Rectum 2014; 57: 694-649 
[184] Little MW, Oakley T, Briggs JH et al. Technical and clinical outcomes following colonic stenting: a seven-year analysis of 268 procedures. Cardiovasc Intervent Radiol 2016; 39: 1471-1478

[185] Matsuda A, Miyashita M, Matsumoto S et al. Comparison between metallic stent and transanal decompression tube for malignant large-bowel obstruction. J Surg Res 2016; 205: 474-481

[186] Mitra V, Hu M, Majumdar D et al. Safety and efficacy of self-expandable metal stents for obstructive proximal and distal large bowel cancer. J R Coll Physicians Edinb 2017; 47: 30-34

[187] van den Berg MW, Sloothaak DA, Dijkgraaf MG et al. Bridge-to-surgery stent placement versus emergency surgery for acute malignant colonic obstruction. Br J Surg 2014; 101: 867-873

[188] Wang FG, Bai RX, Yan M et al. Short-term outcomes of self-expandable metallic stent versus decompression tube for malignant colorectal obstruction: a meta-analysis of clinical data. J Invest Surg 2019: doi:10.1080/08941939.2019.1566419 Epub ahead of print

[189] Yang SY, Park YY, Han YD et al. Oncologic outcomes of self-expandable metallic stent as a bridge to surgery and safety and feasibility of minimally invasive surgery for acute malignant colonic obstruction. Ann Surg Oncol 2019; 26: 2787-2796

[190] Lara-Romero C, Vilches A, Caunedo-Alvarez A et al. Better recurrence-free survival after stent bridge to surgery compared to emergency surgery for obstructive left-sided colonic cancer in patients with stage III status of the American Joint Committee on Cancer (AJCC): a bicentric retrospective study. Int J Colorectal Dis 2019; 34: $1241-1250$

[191] Zanghi A, Piccolo G, Cavallaro A et al. A pilot study about the oncologic safety of colonic self-expandable metal stents (SEMS) in obstructive colon cancer: is occlusion always better than "silent" perforation? Eur Rev Med Pharmacol Sci 2016; 20: 5242-5248

[192] Fernandez-Esparrach G, Bordas JM et al. Severe complications limit long-term clinical success of self-expanding metal stents in patients with obstructive colorectal cancer. Am J Gastroenterol 2010; 105: 1087-1093

[193] Kawachi J, Kashiwagi H, Shimoyama R et al. Comparison of efficacies of the self-expandable metallic stent versus transanal drainage tube and emergency surgery for malignant left-sided colon obstruction. Asian J Surg 2018; 41: 498-505

[194] van den Berg MW, Ledeboer M, Dijkgraaf MG et al. Long-term results of palliative stent placement for acute malignant colonic obstruction. Surg Endosc 2015; 29: 1580-1585

[195] van Hooft JE, Fockens P, Marinelli AW et al. Early closure of a multicenter randomized clinical trial of endoscopic stenting versus surgery for stage IV left-sided colorectal cancer. Endoscopy 2008; 40: 184-191
[196] Suarez ], Marin G, Vera R et al. Stent placement prior to initiation of chemotherapy in patients with obstructive, nonoperative left sided tumors is associated with fewer stomas. J Surg Oncol 2017; 115: 856-863

[197] Lim TZ, Chan DKH, Tan KK. Endoscopic stenting should be advocated in patients with stage IV colorectal cancer presenting with acute obstruction. J Gastrointest Oncol 2018; 9: 785-790

[198] Ahn HJ, Kim SW, Lee SW et al. Long-term outcomes of palliation for unresectable colorectal cancer obstruction in patients with good performance status: endoscopic stent versus surgery. Surg Endosc 2016; 30: 4765-4775

[199] Saeed KM, Zafar W, Masood MA et al. Self-expanding metallic stents (SEMS) in left-sided colonic cancer - a cancer center experience. J Gastrointest Cancer 2016; 47: 69-74

[200] Watt AM, Faragher IG, Griffin TT et al. Self-expanding metallic stents for relieving malignant colorectal obstruction: a systematic review. Ann Surg 2007; 246: 24-30

[201] Meisner S, Gonzalez-Huix F, Vandervoort JG et al. Self-expanding metal stenting for palliation of patients with malignant colonic obstruction: effectiveness and efficacy on 255 patients with 12month's follow-up. Gastroenterol Res Pract 2012; 2012: 296347

[202] Alford T, Ghosh S, Wong C et al. Clinical outcomes of stenting for colorectal obstruction at a tertiary centre. J Gastrointest Cancer 2014; 45: 61-55

[203] Canena JM, Liberato M, Marques I et al. Sustained relief of obstructive symptoms for the remaining life of patients following placement of an expandable metal stent for malignant colorectal obstruction. Rev Esp Enferm Dig 2012; 104: 418-425

[204] Han JP, Hong SJ, Kim SH et al. Palliative self-expandable metal stents for acute malignant colorectal obstruction: clinical outcomes and risk factors for complications. Scand J Gastroenterol 2014; 49: 967973

[205] Padwick RT, Chauhan V, Newman M et al. Endoscopic stenting of acutely obstructing colorectal cancer: a 10-year review from a tertiary referral centre. ANZ J Surg 2016; 86: 778-781

[206] Fiori E, Lamazza A, Sterpetti AV et al. Endoscopic stenting for colorectal cancer: lessons learned from a 15-year experience. J Clin Gastroenterol 2018; 52: 418-422

[207] Luigiano C, Ferrara F, Fabbri C et al. Through-the-scope large diameter self-expanding metal stent placement as a safe and effective technique for palliation of malignant colorectal obstruction: a single center experience with a long-term follow-up. Scand J Gastroenterol 2011; 46: 591-596

[208] Yoshida S, Watabe $\mathrm{H}$, Isayama $\mathrm{H}$ et al. Feasibility of a new selfexpandable metallic stent for patients with malignant colorectal obstruction. Dig Endosc 2013; 25: 160-166 